\title{
SSTP1, A Host Defense Peptide, Exploits the Immunomodulatory II6 Pathway to Induce Apoptosis in Cancer Cells
}

Shyla G.

Rajiv Gandhi Centre for Biotechnology

Soumya Krishnan U.

Rajiv Gandhi Centre for Biotechnology

Amrutha Mohan

Rajiv Gandhi Centre for Biotechnology

Geetha Shanmugam

Rajiv Gandhi Centre for Biotechnology

Vineethkumar T.V.

Rajiv Gandhi Centre for Biotechnology

\section{Sreekumar J.}

CTCRI: Central Tuber Crops Research Institute

Sivakumar K.C.

Rajiv Gandhi Centre for Biotechnology

Dileep Vasudevan

Institute of Life Sciences

Sai Ravi Chandra Nori

Rajiv Gandhi Centre for Biotechnology

Shijulal Nelson Sathi

Rajiv Gandhi Centre for Biotechnology

\section{Sanil George}

Rajiv Gandhi Centre for Biotechnology

Tessy Thomas Maliekal ( $\nabla$ tessy@rgcb.res.in )

Rajiv Gandhi Centre for Biotechnology https://orcid.org/0000-0002-7311-7950

\section{Research}

Keywords: Host Defense Peptides, Temporins, Immunoregulation, IL6 pathway, JNK/AP1 pathway

Posted Date: July 9th, 2021

DOl: https://doi.org/10.21203/rs.3.rs-678241/v1 
License: (c) (i) This work is licensed under a Creative Commons Attribution 4.0 International License. Read Full License 


\section{Abstract}

Background: The anticancer activities of host defense peptides (HDPs) are mainly attributed to their cell penetrating activity. Accordingly, several approaches based on machine learning, calculating the membrane pore formation ability, have been proposed for the anti-cancer peptide identification. Since the membranolytic activity can lead to nonspecific effects, like hemolysis, the therapeutic application of such molecules is limited. In this context, we considered the immunomodulatory activity of HDPs, which is regulated by specific membrane targets and immunomodulatory pathways in immune cells. As many immunomodulators are aberrantly expressed in cancer cells, the possibility of the activation of an immunomodulatory pathway was investigated for a novel anticancer HDP, SSTP1.

Methods: The fourteen mature peptides identified by shotgun cloning from the frog-skin secretions were screened for cytotoxicity in oral cancer cells. The mechanism of action of the selected peptide, SSTP1 was investigated in comparison to its inactive mutant, SSTP2. An RNA-Seq coupled with pathway enrichment analysis was performed to identify the upstream signaling leading to the mitochondrial pathway of apoptosis. Since the activation of the IL6/IL6R pathway was suggested, we performed in silico docking studies to find the binding of SSTP1 to the IL6/IL6Ra/gp130 complex. The dynamic simulation predicted the conformational changes in the active site residues. The confocal co-localization studies, pull-down assay, FRET analysis, western blot and reporter assays were performed to elucidate the role of the IL6/IL6R pathway in SSTP1-induced apoptosis. Specific small molecule inhibitors and neutralizing antibodies were used to ascertain the role of the IL6/IL6Ra/gp130 complex in the activation of JNK/AP1 pathway-dependent cell death.

Results: SSTP1, a novel temporin, modulates the IL6 pathway and induces apoptosis when it binds to IL6Ra on the active IL6/IL6Ra/gp130 complex, rearranging the active site residues. In contrast to the IL6 blockers inhibiting JAK/STAT activity, SSTP1 shifts the proliferative IL6/JAK/STAT signaling to the apoptotic IL6/JNK/AP1 pathway. In IL6Ra-overexpressing cancer cells, apoptosis is preferred over the membranolytic activity, upon SSTP1 treatment.

Conclusions: Here, we provide the evidence of an HDP-induced signaling through immunomodulators, leading to apoptosis in cancer cells. Our study also implies the importance of identifying the targets of HDPs for their clinical application

\section{Background}

HDPs isolated from amphibians and marine organisms have been widely investigated for their antitumor activity based on their antimicrobial activity. Since cancer cell membrane has a high negative charge on the outer surface due to aberrant expression of anionic molecules, majority of the reports show the basis of antitumor property as membranolytic activity, similar to the bacterial membrane pore formation [1]. HDPs kill bacteria by disrupting the bacterial membrane via pore formation in the lipid membrane, thinning of the membrane bilayer, membrane dissolution, or lipid-peptide domain formation [2]. The 
characteristics required for this membranolytic activity is used for predicting the anticancer property of peptides and several algorithms are developed for this recently $[3,4,5]$. But the non-specific membranolytic activity limits the therapeutic use of antitumor HDPs, as it leads to hemolysis and other side effects $[2,6,7]$ However, the induction of apoptosis might depend on mechanisms other than the regulation of innate immunity and membranolytic activity, involving specific cell signaling [1]. Although some HDPs are reported to induce apoptosis depending on the modulation of ROS production and the activation of the mitochondrial pathway [8], the upstream signaling pathways remain largely unknown [9, 10].

Host defense peptides (HDPs), thought initially as the antimicrobial peptides are now considered as immunomodulators because of their multi-faceted roles in the host's innate and adaptive immunity. Certain immunomodulatory activities are also reported for HDPs, like the stimulation of cytokines and chemotaxis for leukocytes [6]. Apart from these functions, some of the human HDPs, like Cathelicidin, aDefensins and $\beta$-Defensins are shown to modulate tumor properties [7]. Some of the evidences from Cathelicidin null mice reinforce the role of HDPs in tumor suppression [11]. Over-expression of a Cathelicidin, LL-37, suppresses tumorigenesis in colon and gastric cancer cells [12]. LL-37 interacts with $N$-formyl peptide receptor like 1 (FPRL1) on immunomodulators $[13,14]$, increasing their cytotoxic effects on cancer cells, leading to tumor suppression [12]. In contrast, LL-37 promotes tumor progression in some contexts, depending on its binding to FPR2 and P2X ${ }_{7} R$ in pancreatic cancer [15] and IGF-1R in breast cancer [12]. Nevertheless, the mechanism by which LL-37 induces apoptosis in cancer cells of different origin is not clearly understood $[7,16]$. In parallel, several other HDPs of nonhuman origin also induce apoptosis in cancer cells independent of the innate immune regulatory mechanisms [1].

Recalling the relevance of FPRL1 for LL-37, it can be speculated that the upstream signaling pathway initiated by HDPs could be one of the immunomodulatory pathways, which leads to apoptosis. Here, we attempted to elucidate the mechanism by which one of the HDPs, identified from the skin secretion of an indigenous frog, induces apoptosis in oral cancer cells. The present study suggests that the immunomodulatory mechanism exerted by HDPs on cancer cells leads to cell death.

\section{Methods}

\section{Cells and reagents used}

HSC-4 and RCB1015 cells were collected from RIKEN BRC Cell Bank, Japan. CaSki, MDA-MB-231, SiHa, HeLa and A-375 were obtained from the RGCB cell repository. The following antibodies for IL6Ra, DSG-3, $\beta$ Tubulin, a5 tubulin, $\beta$ - actin, GRPR, Shh, DLL, PARP-1, STAT 3, JNK 1, c-JUN and Rab5C were purchased from Santacruz Biotechnology Inc, USA. Antibodies for Caspase 9, Caspase 8, Caspase 3, Caspase 7, phospho-STAT3, phospho-STAT3, phospho-JNK, phospho-C-JUN, STAT1, phospho-STAT1, phosphoMAPK1/2 and phospho-AKT, were purchased from Cell Signaling Technology, USA. Secondary antibodies, Peroxidase conjugated AffiniPure Donkey Anti Mouse, AffiniPure Donkey Anti Goat and AffiniPure Donkey Anti-Rabbit Secondary Antibodies were from Jakson ImmunoResearch Laboratories Inc, USA. Donkey 
Anti-Goat Alexa Fluor-568, Donkey Anti-Mouse Alexa Fluor-568, Donkey Anti-Rabbit Alexa Fluor-568, Donkey Anti-Goat Alexa Fluor-680 and Streptavidin Alexa Flour-488 (Molecular Probes, Life Technologies) were used for immunofluorescence.

SSTP1 (FLPLLISALTSLFPKLGK) and SSTP2 (FLPRRISARTSLFPKRGK- $\mathrm{NH}_{2}$ ) were synthesized from SynPeptide Co. Ltd, Shanghai, China. Peptides were labeled with D-Biotin Succinimidyl ester (Life Technologies) or Alexa Flour-488 (Life Technologies). 3xAP1pGL3 was a gift from Alexander Dent (Addgene plasmid\#40342; http://n2t.net/addgene:40342; RRID:Addgene 40342). MitoTracker Deep Red (Thermo Fisher Scientific) was used for imaging. S-Ruxolitinib (Selleckchem), SP600125 (InvivoGen) and LMT- 28 (Sigma Aldrich) were the inhibitors used for the study. Dynabeads M-280 Streptavidin (Invitrogen) was used for pull-down assay.

\section{Screening of cDNA encoding peptides through shotgun cloning}

cDNA library constructed from frog skin secretions (10) was subjected to RACE amplification with a sense (5'ATGTTCACCTTGAAGAAT) and anti-sense primer (5'- AGATGATTTCCAATTCCAT-3') Purified amplicons were cloned into pGEMT-easy vector (Promega) and positive clones were amplified and sequenced (ABIDNA sequencer 3730). The nucleotide sequences were translated to amino acid sequence with EMBOSS Transeq (http://www.ebi.ac.uk/Tools/st/emboss_transeq/) and were subjected to homology searches using NCBI BLAST (http://w.w.w.ncbi.nlm.nih.gov). Antimicrobial peptide database (APD, http://aps.unmc.edu/AP/main.php) was used for peptide prediction. Physical parameters were computed using ProtParam (http://expasy.org/tools/protparam.html).

\section{MTT and IC- 50 calculation}

Concentration- and time-dependent effects on cell growth inhibition were measured using the MTT [3-(4,5dimethylthiazol-2-yl) - 2,5-diphenyltetrazolium bromide] assay. 5000 cells/well were seeded in 96-well plate and were treated with peptides for $48 \mathrm{~h}$. Cells were incubated with MTT $(1.25 \mathrm{mg} / \mathrm{ml})$ for 2 hours at $37^{\circ} \mathrm{C}$. The blue formazan crystals were solubilized in isopropanol and read at $595 \mathrm{~nm}$.

\section{Annexin/ PI staining}

The cells were stained with Annexin V apoptosis detection kit (Santacruz Biotechnology, Inc), following the instructions of manufacturer and analyzed using BD FACS Ariall.

\section{Western Blotting}

Western blotting was performed as reported earlier [17]. Total proteins extracted from cells were resolved on SDS-PAGE, transferred onto nitrocellulose membranes, which were incubated with corresponding primary antibody followed by horseradish peroxidase-linked secondary antibody. The images were developed using Clarity western ECL substrate (Bio-Rad) and images were captured on X-Ray sheet. Densitometric Analysis was performed using software ImageJ $1.52 \mathrm{v}$. the average and standard deviation of fold change based on control from biological replicates were plotted . 


\section{Immunofluorescence staining and confocal microscopy}

HSC-4 cells were grown overnight on confocal dish. Immunofuorescence was performed as described [17]. The images were acquired in Nikon A1R LSCM or Leica SP8 WII Confocal Microscope.

\section{Co-localization studies}

The images were acquired in Nikon A1R LSCM confocal microscope with 60X objective, oil immersion and the analysis was performed using NIS-Elements AR 4.00.04.. For the co-existence of SSTP1 and transferrin, SSTP1 was labeled with Alexa Fluor-488 and transferrin with Alexa Fluor-568. The imaging was performed with a pinhole size of $39.7 \mu \mathrm{m}$ giving an optical resolution of $0.10 \mu \mathrm{m}$ for Alexa Fluor-488 and $0.12 \mu \mathrm{m}$ for Alexa Fluor-568. The Mander's overlap was calculated. For analyzing the physical interaction of SSTP1 and IL6Ra, Biotin-labeled SSTP1 $(10 \mu \mathrm{M})$ was added to cell and incubated for 30 minutes. Cells were washed, fixed, blocked with $2 \%$ FBS and probed for IL6Ra using antibody for extracellular domain. The signals were visualized using streptavidin-488 and anti-mouse Alexa Fluor-680. The imaging was performed with a pinhole size of $43.2 \mu \mathrm{m}$ giving an optical resolution of $0.10 \mu \mathrm{m}$ for Alexa Fluor-488 and $0.13 \mu \mathrm{m}$ for Alexa Fluor-680. The co-localization indicating a physical interaction was calculated as Pearson's co-efficient. To study the endosomal co-localization of SSTP1 and IL6Ra, the experiment was performed as before. Streptavidin-Alexa Fluor-488, anti-mouse Alexa Fluor-568 and antigoat Alexa Fluor-680 were used for visualization. The imaging was performed with a pinhole size of 43.2 $\mu \mathrm{m}$ giving an optical resolution of $0.10 \mu \mathrm{m}$ for Alexa Fluor-488, $0.12 \mu \mathrm{m}$ for Alexa Fluor-568 and $0.13 \mu \mathrm{m}$ for Alexa Fluor-680. Mander's overlap was calculated to analyze the co-existence. In all cases, single staining was performed to rule out the bleed through.

\section{FRET analysis}

For the FRET analysis, Alexa Fluor-488 and Alexa Fluor-555 was used as the FRET pair. Cells were treated with biotin-labeled SSTP1 $(10 \mu \mathrm{M})$ for 30 minutes, unbound peptides were washed off and cells were fixed and stained for IL6Ra. To visualize staining streptavidin-Alexa Fluor-488 and anti-mouse Alexa Fluor-555 were used. The images were acquired using Olympus FV3006 in FRET mode. The image analysis was performed using FV31S-SW software.

\section{RNA isolation, Library preparation and Sequencing}

RNA was isolated from the cells using RNeasy mini kit (Qiagen). mRNA-Sequencing libraries were prepared using NEBNextR Ultra ${ }^{\mathrm{TM}}$ II RNA Library PrepKit for IlluminaR (NEB) following the manufacturer's instructions. The sequence data quality was checked using FastQC and MultiQC software. The QC passed reads were mapped onto indexed Human reference genome (GRCh38.p7) using TopHat2 and were annotated with Human GTF (GRCh38) downloaded from GENCODE. Transcripts were assembled using Cufflinks. The assembled transcripts of the samples were merged together using the Cuffmerge. Cuffcompare was used to compare the assembled transcripts with GENCODE, annotation. Differential gene expression analysis was carried out using Cuffdiff. The genes that showed significant differential expressions were used for pathway enrichment analysis by WebGestalt. 


\section{RT-PCR and qRT-PCR}

Total RNA was extracted and reverse transcribed using Maxima Reverse transcriptase (Thermo Scientific). RT-PCR or qRT-PCR was done to assess gene expression with gene-specific qRT-PCR primers (Supplementary Table 4). RT-PCR was done using Master cycler Gradient PCR (Eppendorf). qRT-PCR reactions were done in 7500 Real-time PCR System (Applied Biosystems) using the Maxima SYBR green qPCR master mix; (Thermo Fisher).

\section{Pull-down Assay}

HSC-4 cells were lysed with immunoprecipitation buffer [0.025M Tris, 0.15 M Sodium chloride, $0.001 \mathrm{M}$ EDTA, $0.5 \%(\mathrm{v} / \mathrm{v})$ NP40, $0.5 \%(\mathrm{v} / \mathrm{v})$ Triton $X 100,5 \%$ glycerol $(\mathrm{v} / \mathrm{v}), 1 \times$ protease inhibitor, $\mathrm{pH}$ 7.4]. For affinity pull-down, $10 \mu$ mols of biotin-labelled peptides were incubated with cell lysate containing $1 \mathrm{mg}$ of protein, for 1 hour at $4^{\circ} \mathrm{C}$ followed by overnight incubation with streptavidin Dynabeads. After washes, bound peptide-protein complexes were separated from the beads by boiling in $5 \times$ dye, resolved on $10 \%$ SDS-PAGE and were used for western blot analysis.

\section{Docking and Dynamic simulation}

SSTP1 and SSTP2 structures were modeled individually using the PEP-FOLD3 peptide structure prediction server [18] by biased model approach with default settings, using the structure of Temporin-1 Ta (PDB id: 2MAA). The best models of SSTP1 and SSTP2, as suggested by the server were chosen. Docking of SSTP1 and SSTP2 were done with IL6/IL6Ra/gp130 complex (PDB No.1P9M) using ClusPro and docking scores were calculated. Structures with highest scores were further used for simulation studies. The structures obtained from the molecular docking were subjected to molecular dynamics simulation using GROMACS version 5.0.4 with all-atom/OPLS-AA force field. The docked models were solvated in a periodic cubic box containing flexible, simple point charge (SPC) water molecules and neutralized with $\mathrm{Na}+$ and $\mathrm{Cl}$-counter ions replacing the water molecules. The particle mesh Ewald method was used for electrostatic interactions of the simulation system periodic boundary conditions with grid dimensions of $1.0 \AA$ and a $12.5 \AA$ cut-off for Van der Waals interaction. The prepared systems underwent minimization (5000 steps of steepest descent and 5000 steps of conjugated gradient) and further subjected to equilibration at a temperature $300 \mathrm{~K}$ and a pressure 1 bar for a total of $10 \mathrm{~ns}$. Finally, the systems were subjected to MD simulations for $100 \mathrm{~ns}$, and the atomic coordinates were recorded every 10 ps for further analysis. Comparative structural deviations in systems were analysed with the coordinates of the vectors generated during MD simulations after obtaining hierarchal MD clusters. Interface area ( $\AA 2$ ) and hydrogen-bonds were calculated using the PISA server.

\section{Reporter Assay}

HSC-4 cells (50,000 cells/well) were seeded in a 24-well plate, and 3×AP1pGL3 [19] and Renilla luciferase plasmids were transfected in 10:1 ratio using Lipofecatmine2000 (GIBCO). Cells were treated with the peptides, and cell lysates were prepared to perform luciferase assay using Dual Luciferase Reporter Assay system (Promega), following manufacturer's instruction. 


\section{Hemolytic Assay}

Hemolytic assays of SSTP1 was carried out as described [20]. Briefly, fresh human RBCs were resuspended in PBS to make $4 \%(\mathrm{v} / \mathrm{v})$ solution and exposed to serially diluted peptides in PBS at $37^{\circ} \mathrm{C}$. After $1 \mathrm{~h}$, the amount of hemoglobin released was estimated by measuring the absorbance of the supernatant at $415 \mathrm{~nm}$. Hemolysis with Triton X-100 was used as the positive control.

\section{Statistical Analyses}

$\mathrm{IC}_{50}$ value for HSC-4 cells and MDA-MB-231 cells were calculated with Dose-response model. Analysis of dose-response data is made available through the 'drc' package in $\mathrm{R}$ environment for statistical computing. The "drm" function in the package "drc" is a general model fitting function for analysis of concentration/dose/time-effect/response data and the four parameter a log-logistic model was fitted for estimating the slope, lower, upper and $\mathrm{IC}_{50}$ values. All the other data analyses were performed using GraphPad Prism 8.4.3. The line graphs were prepared using non-linear curve fit-four parameters. The statistical significance between two groups was analyzed using Students paired two-tailed t-test.

\section{Results}

\section{Identification of antitumor peptides from the skin secretion of Indosylvirana aurantiaca}

Skin secretion from Indosylvirana aurantiaca was used to construct a cDNA library by shotgun cloning [20]. The 14 mature peptides identified from the frog's skin secretion (Supplementary Table 1) were chemically synthesized to check the antitumor activity. One of the peptides, Temporin 1IDau1, was chosen for further analysis due to its low $\mathrm{IC}_{50}$ value. This peptide is referred to as SSTP1 in our studies. The physicochemical properties of SSTP1 are listed in Supplementary Table .2. The helical wheel projection of SSTP1 showed a spatial separation of hydrophilic residues and hydrophobic residues imparting amphipathicity (Supplementary Fig. 1). We modified this peptide sequence by replacing the hydrophobic residues with Arginine to generate a control peptide, SSTP2, with less helicity and amphipathicity. Thus, the in silico analysis predicted SSTP2 to be a negative control for the potent antitumor peptide, SSTP1.

\section{SSTP1 induces apoptosis in oral cancer cells}

In HSC-4 cells, SSTP1 induces growth inhibition with an IC $\mathrm{C}_{50}$ value of $10.22 \mu \mathrm{M}$, while SSTP2 has negligible cytotoxicity (Fig. 1a). We performed Annexin/PI staining, and the population with Annexin alone (early apoptotic), Annexin/PI dual stained (late apoptotic), and PI alone (dead cells) were quantified by FACS. We observed a 3-fold increase in the early apoptotic population upon SSTP1 treatment (Fig. 1b and c). Further analysis showed the active cleavage of PARP, Caspase 3, 7, and 9, while Caspase 8 was unaffected by SSTP1 treatment. The cells treated with SSTP2 neither showed an increase in apoptotic population nor activation of any of the Caspases (Fig. 1d and e). As all these evidences suggested the 
involvement of mitochondrial pathway of apoptosis, we explored the upstream regulators of this pathway.

\section{Induction of apoptosis by SSTP1 involves a non-membranolytic activity}

Our live imaging showed that SSTP1 is binding to the membrane, and it starts internalizing within 30 min. Though at a lower rate, SSTP2 was also binding to the membrane and internalizing. When we repeated the experiments on ice, where cells are not physiologically active, SSTP1 was not appreciably internalized as observed at $37^{\circ} \mathrm{C}$, while internalization of SSTP2 was not affected (Fig. 2a). Since these results suggested the involvement of a receptor-mediated internalization for SSTP1, we decided to check whether it is clathrin-dependent mechanism, using labeled transferrin (Fig. 2b, c and d). Since SSTP1 was not internalized along with transferrin, as suggested by the negligible Mander's overlap, the entry of SSTP1 is probably through receptor-dependent clathrin-independent endocytosis. We further investigated the genes and pathways activated by SSTP1 treatment, which might give insight to its membrane receptors.

\section{RNA-Seq analysis suggests the involvement of the IL6/IL6R pathway in SSTP1-induced apoptosis}

An RNA-Seq (RNA sequencing) on the Illumina platform was performed to identify the target genes of SSTP1, using cells treated with SSTP1 in comparison to SSTP2. Out of the 34 to 37 million reads obtained, 31 to 34 million reads were mapped onto the indexed Human reference genome GRCh38.p7. The differential gene expression analysis identified 208 up-regulated genes and 118 down-regulated genes (Fig. 3a and b). The RNA-Seq results were validated by 12 up-regulated genes and four downregulated genes by RT-PCR and q-PCR (Fig. $3 \mathrm{c}$ and d). Several of the differentially expressed genes were related to apoptosis, (Supplementary Table 3), and the most significant pathway regulated was cytokine signaling (Fig. 3e). There were interleukins and their receptors along with other regulators of cytokine signaling (Fig. 4a). Figure 4b and Supplementary Fig. 2 represent a possible cytokine signaling pathway that leads to apoptosis upon SSTP1-treatment.. Molecules like DUSPS and SOCS1, which regulate STAT and MAPK functions, respectively, were up-regulated by SSTP1 treatment. There was a notable upregulation of AP1 subunits, JUN and FOS. Since the ligand, receptor and intermediates for the IL6/IL6R pathway were up-regulated, we focused on this pathway. Yet, the pro-tumorigenic targets of the JAK/STAT pathway, like CyclinD1, cMyc and PIM were not regulated.

\section{SSTP1 binds to IL6Ra on the cell membrane}

To check whether SSTP1 activates the IL6/IL6R pathway by binding to IL6Ra, we checked the colocalization of SSTP1 and IL6Ra on cell membrane. We observed several foci on the cell surface with colocalization of SSTP1 and IL6Ra, while co-localization of SSTP2 to IL6Ra was rare as shown by the Pearson's coefficient (Fig. 5a and b). In order to rule out a nonspecific interaction of SSTP1 to a random surface receptor, we performed a co-immunofluorescence analysis of SSTP1 with several surface molecules like tubulin a5, $\beta$-tubulin, DSG3, DLL1 and GRPR, which did not show co-localization with the peptide (Supplementary Fig. 3). To visualize the IL6R-dependent SSTP1 endosomal up-take, we used an early endosome marker, Rab5C. We observed the co-existence of SSTP1 and IL6Ra within early endosomes, marked by Rab5C (Fig. 5c and d). The physical interaction of SSTP1 with IL6Ra was 
stronger than that of SSTP2, as revealed by the pull-down assay using biotin-labeled peptides (Fig. 5e). The physical interaction of SSTP1 and IL6Ra was also ascertained using FRET analysis as described under Methods (Fig. 5f).

\section{In silico analyses suggest that SSTP1 and SSTP2 exert differential effects on active sites of IL6/IL6Ra/gp130 complex}

To get insight into dynamics of peptide binding, we performed some in silico analyses. As the peptides' crystal structures were not known, we used the 3D structure of another peptide from the same family, Temporin-1 Ta, as a template to predict the structures of SSTP1 and SSTP2 (Supplementary Fig. 4). Since signaling depends on the formation of the active complex of IL6 and its receptors, we docked the peptides to the reported crystal structure of the active hexameric complex. The analysis predicted stronger interaction for SSTP1 than SSTP2 (with scores - 985.038 and - 789.990 for SSTP1 and SSTP2, respectively). Dynamic simulations for the structures were performed, and the resulting stable conformations with the least energy were analyzed further. Both SSTP1 and SSTP2 bind to the interface between IL6 and IL6Ra, but in a different orientation (Fig. 6a).

The superimposition of the peptide-bound complexes to the unbound complex showed that a unique rearrangement occurs in the IL6/IL6R interface of SSTP1-bound complex, specifically in a region that forms an interface between gp130 of the other trimer (Fig. 6b). The hydrogen bonds formed between Arg30 and Glu297; Arg182 and Ser247; as well as Lys54 and GIn209 were disrupted when SSTP1 or SSTP2 binds to the complex (Fig. 6c). On further analysis, it was found that the significant unique change observed in the SSTP1-bound complex is in the interface region constituted by Phe153 and Asn155 of IL6Ra and Lys54, Glu55, Ala56, Leu57 and Asn61 of IL6 (Fig. 6c). There was a difference in the values for accessible surface area, buried surface area and solvation energy for these residues in SSTP1bound 1P9M in comparison to 1P9M and SSTP2-bound 1P9M, so that the conformation of these residues was significantly altered.

\section{SSTP1 down-regulates JAK/STAT and MAPK pathways activating JNK/AP1 pathway}

There are evidences to show that the IL6/IL6R pathway leads to the activation of JAK/STAT, JNK/AP1, MAPK and PI3K/NF-KB pathways [21, 22, 23, 24]. The phosphorylated forms of STAT1 (Y701) and STAT3 (Y705) were probed to check the JAK/STAT pathway activation. Another phosphorylation of STAT3 at S727, which is reported to be independent of JAK activation [25], was also probed. Even though SSTP1 did not affect STAT1a (Y701) phosphorylation, it drastically reduced STAT3 (Y705) phosphorylation along with an increase in PSTAT3 (S727) levels. On the other hand, SSTP2-treatment did not significantly alter the phosphorylations of STAT1 $a$ or STAT3 (Fig. 7a and b). SSTP1 markedly up-regulated phosphorylation of JNK1 and pcJUN compared to SSTP2. Though both the peptides down-regulated pMAPK levels, pAKT levels were unaffected. All these biochemical analyses point out that SSTP1 preferentially down-regulates JAK-mediated-phosphorylation of STATs and MAPK, simultaneously upregulating phosphorylation of JNK1 and c-JUN. A luciferase AP1 reporter assay showed that AP1 is activated by SSTP1 in comparison to SSTP2 (Fig. 7c). 


\section{SSTP1-mediated induction of JNK /AP1 pathway depends on IL6/IL6Ra/gp130 complex}

Inhibitors for IL6 (LMT-28), JAK (S-Ruxolitinib) and JNK (SP600125) were used to check the dispensability of each of these molecules in activating cJUN by SSTP1. The addition of these inhibitors blocked the phosphorylation of STAT1 and STAT3 (Fig. 8a and b). The SSTP1-mediated up-regulation of pJNK was not appreciably affected by either of these inhibitors, while pcJUN up-regulation was inhibited by only SP600125 (Fig. 8a and b). At the same time, SC-144, the inhibitor for gp130, and an antibody for IL6Ra significantly reduced phosphorylation of STATs, JNK and cJUN (Fig. 8c and d). The SSTP1-induced growth inhibition of cancer cells was significantly inhibited by anti-IL6Ra and SC144, whereas LMT28 was ineffective (Fig. 8e)

\section{Growth inhibition by SSTP1 depends on IL6Ra levels}

The evaluation of the mechanism of induction of growth inhibition by SSTP1 showed that it depends on the modulation of IL6/IL6R pathway by binding to IL6Ra. This suggested that the peptide might have better growth inhibitory property on IL6Ra-overexpressing cell lines. Since triple-negative breast cancer, prostate cancer and lung cancer express high level of this molecule, we investigated the growth inhibitory property of SSTP1 on these cell lines. The up-regulation of IL6Ra expression was confirmed (Fig. 9a and b). As we expected, there was a drastic increase in the growth inhibitory potential for SSTP1 in these cells (Fig. 9c). Notably, there was $91 \%$ growth inhibition for triple-negative breast cancer cell line MDA-MB-231. At the $\mathrm{IC}_{50}$ concentration $(4.5 \mu \mathrm{M})$ of this cell line, SSTP1 induced activation of JNK and phosphorylation of c-JUN, while the level of pSTAT3 was extremely low in treated and untreated cells (Fig. 9d, e and f). Although many of the bioactive peptides induce hemolysis, SSTP1-induced hemolysis at the $\mathrm{IC}_{50}$ concentration was negligible (Fig. 9d).

\section{Discussion}

Temporins are a class of HDPs with antibacterial and antitumor activities, reported from a wide range of frogs of the Ranidae family [26]. A few of the family members like Temporin-1CEa, TemporinA, and Temporin-SHa are shown to have antitumor activities against certain cancer types $[27,28,29]$. The selective cytotoxicity of TemporinA towards lung cancer cells is attributed to the specific phospholipid composition of lung cancer cells [27]. The anti-cancer property of Temporin-1CEa depends on both membranolytic activity and mitochondrial pathway of apoptosis [29]. Peptides, like temporins with high hydrophobicity, mild amphipathicity, and helical structure, are reported to have antitumor activities [30]. While SSTP1 satisfies these conditions, SSTP2 had no amphipathicity as the hydrophobic Leucine residues were replaced by positively charged Arginine, serving as a negative control for SSTP1. As reported for other temporins, SSTP1 also induced apoptosis by mitochondrial pathway [28, 29]. Even though induction of apoptosis by HDPs is known, their upstream signaling pathways and the molecular targets for most of them are not evaluated yet.

The differential expression of various cytokines and their intermediates in our RNA-Seq analysis substantiated their role in immunomodulation, which is required for host defense peptides. The 
suppressor of cytokine (SOCS) are important negative regulators of JAK-dependent cytokine signaling [31]. The SSTP1-dependent up-regulation of SOCS1 clearly indicated the suppression of cytokinemediated JAK/STAT signaling. Our pathway analysis suggested the activation of the cytokine signaling pathway, possibly downstream of IL6Ra. This is in accordance with our earlier observation of the mode of internalization, since IL6Ra is known to be internalized through clathrin-independent pathway [32]. Further, we also confirmed the physical interaction of the peptide with IL6Ra, reinforcing the importance of IL6/IL6R signaling pathway in SSTP1-induced apoptosis. Although immune activation by chemotaxis and cytokine release are hall marks of HDPs[6], certain temporins are reported to have anti-inflammatory roles by the modulation of cytokine pathway [33,34]. In pseudomonas-infected bronchial cells, TemporinB induces pro-inflammatory responses, while its analogue induces anti-inflammatory responses [35]. L-K6, an analog of temporin-1CEb, suppresses the cytokine signaling pathways in LPS-stimulated macrophages [34]. In an altered context, like cancer, the peptide induces apoptosis, probably utilizing similar signaling pathways.

Upon binding to IL6Ra and gp130, IL6 activates different signaling pathways like JAK/STAT, PI3K/NF-KB, Ras/MAPK and JNK/AP1 pathways, depending on the context $[21,22,23,24]$. Consistent with the upregulated SOCS1, we did not observe the classical proliferative JAK/STAT downstream molecules like CyclinD1, cMYC and PIM [36, 37]. We observed an up-regulation of DUSPs in our RNA-Seq analysis, which are the negative regulators for MAPK signaling $[38,39]$. Another up-regulated molecule was an E3 ubiquitin ligase, $K L H L 21$, which is reported to block NF-KB activity by targeting IKK $\beta$ [40]. JNK/AP1 pathway is generally a survival pathway, but in certain instances it is shown to induce apoptosis [41, 42]. Thus the RNA-Seq results suggested that SSTP1 preferentially up-regulates JNK/AP1 pathway by blocking other arms of IL6/IL6R signaling to facilitate apoptosis. The biochemical analysis for the major intermediates of STATs, JNK, MAPK and PI3K pathways revealed the specific activation of JNK/AP1 pathway, consistent with our prediction from RNA-Seq results. The abrogation of the SSTP1-mediated cJun phosphorylation and cytotoxicity upon inhibition of IL6Ra and gp130 strengthened our argument that the AP1 activation depends on the binding of SSTP1 to the active IL6/IL6Ra/gp130 complex.

Among the inhibitors used, LMT-28 blocks the interaction of IL6 with gp130 in the IL6/IL6Ra/gp130 complex [43]. S-Ruxolitinib blocks the JAK-mediated phosphorylation of substrates like STATs, and SP60012 blocks the substrate phosphorylation of JNK $[21,44,45]$. SC144 is the inhibitor for gp130 and an antibody to the extracellular domain of IL6Ra was used to block the IL6Ra activity. All these inhibitors are considered as IL6 blockers as all of them ultimately blocks STAT activation. Our results showed that none of these molecules can activate AP1 pathway. At the same time, SSTP1 effectively induces cytotoxicity and apoptosis by the activation of IL6R/JNK/AP1 pathway with concomitant downregulation of IL6-mediated STAT and MAPK signaling, which are pro-tumorigenic pathways $[43,46,47]$. Thus it is evident that the mechanism of SSTP1-induced cell death is different from the conventional IL6 blockers that inhibit STAT activation alone.

Binding of IL6 to IL6Ra leads to their interaction with gp130, forming a trimeric complex, which in turn dimerizes to form a hexamer [48]. In this conformation, gp130 interacts with the IL6/IL6Ra interface of 
both trimers [48]. The conformational change occurring in the extracellular domain of gp130 leads to the autophosphorylation of gp130, and signal transduction through its cytoplasmic domain. Though both the peptides can bind to the active receptor complex, docking analysis predicted a weaker interaction of SSTP2 compared to SSTP1, which was verified by the absence of co-localization in our imaging, and lesser bound peptide in the pull-down experiments. SSTP1 binds to the active receptor complex between IL6 and IL6Ra, significantly rearranging the active site residues that influence downstream signaling. Detailed analysis of the IL6/IL6Ra interface residues in the peptide-bound and unbound active receptor complex showed a unique rearrangement of residues. The conformations of residues Lys54, Glu55, Ala56 and Leu57 are altered differentially when SSTP1 or SSTP2 binds to the complex, to give a unique conformation for SSTP1-bound active receptor complex. Among these residues, Lys54, Glu55 and Leu57 are reported to have an essential function in IL6-dependent activities [49]. Lys54 and Glu55 are critical for the binding of IL6 to IL6Ra, while Leu57 is required for its binding to gp130 of the other trimer [49]. When these residues were mutated, IL6-dependent proliferative activity of A375 cells was shown to be reduced, wherein mutation of Leu57 had the maximum effect [49]. So our results show that the residues interacting at the interface of IL6 and gp130 from the other trimer are altered upon SSTP1 binding, which might alter the conformational changes induced in gp130 strand upon binding to the trimer. This change in gp130 could further alter the substrate specificity of the downstream signaling. Among the inhibitors used, LMT-28 also binds to the same active receptor complex, but at a different site i.e. the IL6/gp130 interface, probably explaining the differential outcome of SSTP1 and LMT-28. Since there is no competitive binding for these two molecules, LMT-28 fails to block the activity of SSTP1, as we observed in our analyses. In MDA-MB-231 cells, SSTP1-induced cell death primarily depends on the activation of JNK, implying the SSTP1-induced JNK activation is sufficient to induce cell death in IL6Raoverexpressing cancer cells.

\section{Conclusions}

Amphipathic peptides exhibit membranolytic activity, which increases proportionally to the concentration. When the cells express high levels of IL6Ra, SSTP1 binds to the receptor, and acts preferentially through the JNK-dependent mechanism rather than the membranolytic activity, leading to high specificity, as evident from the low $\mathrm{IC}_{50}$ that do not induce hemolysis. Thus, identification of the target of the HDPs helps to identify the sensitive cell types, and achieve the antitumor activity without eliciting the nonspecific side effects. Our study emphasizes the potential of antitumor HDPs with specific membrane targets for clinical translations, since it induce target specific apoptosis, alleviating the side effects.

\section{Abbreviations}

HDP- host defense peptide, RNA-Seq -RNA sequencing

\section{Declarations}


The protocols of the hemolytic assay with human blood samples were approved by the human ethics committee of Rajiv Gandhi Centre for Biotechnology, Thiruvananthapuram, (IHEC/01/2017/20). Blood samples were collected from volunteers after getting the informed consent.

The skin secretions from I. aurantiaca were collected from the hilly terrains of Thiruvananthapuram, Kerala, under a permit from the Kerala Forest Department (WL10-31740).

\section{Consent for publication}

Not applicable

\section{Availability of data and materials}

All the peptides generated during shot gun cloning in this study are included in this published article as Supplementary Table 1.

The datasets generated by RNA-Seq during the current study are available in the GEO repository https://www.ncbi.nlm.nih.gov/geo/query/acc.cgi?acc=GSE173131

\section{Competing interests}

The authors declare that they have no competing interests

\section{Funding}

This work was financially supported by Kerala State Council for Science Technology and Environment, under VK Young Scientist Scheme (717/2014/KSCSTE). Amrutha Mohan acknowledges the financial support from University Grant Commission, India (19/06/2016 (1) EU-V/318501).

\section{Authors' contributions}

Shyla G conceived the idea, conducted the experiments, acquired and analyzed the data. SKU and AM performed the blotting experiments, GS did the reporter assay, SJ did the statistical analysis, VTV and SG contributed to the peptide identification, SKC did the docking and simulation analyses, DV did the peptide structure predictions, SR and SNS did the RNA-Seq. data analysis, and TTM conceived the idea and prepared the manuscript.

\section{Acknowledgments}

Shyla G is thankful to the Director, Rajiv Gandhi Centre for Biotechnology, for providing facilities for conducting the work. 3×AP1pGL3 (3×AP-1 in pGL3-basic) was a gift from Alexander Dent (Addgene plasmid\# 40342; http://n2t.net/addgene:4032. We express our sincere gratitude to T.R. Santhosh Kumar, K. Santhosh Kumar, Ruby John Anto and E. Sreekumar, K.B. Harikumar, Jackson James, Rashmi Mishra, P.K. Umasankar and Suparna Sengupta for providing reagents for our work. We sincerely thank the technical support offered by the members of FACS and Confocal 
Supplementary materials available

\section{References}

1. Gaspar, D., Veiga, A.S., and Castanho, M.A. From antimicrobial to anticancer peptides. A review. Frontiers in microbiology 2013; 4: 294.

2. Oren, Z., and Shai, Y. Mode of action of linear amphipathic a-helical antimicrobial peptides. Peptide Science 1998; 47(6): 451-63.

3. Basith, S., Manavalan, B., Shin, T.H., Lee, D.Y., and Lee, G. Evolution of Machine Learning Algorithms in the Prediction and Design of Anticancer Peptides. Current protein \& peptide science 2020; 21(12): 124250 .

4. Charoenkwan, P., Chiangjong, W., Lee, V.S., Nantasenamat, C., Hasan, M.M., and Shoombuatong, W. Improved prediction and characterization of anticancer activities of peptides using a novel flexible scoring card method. Scientific reports 2021;11(1): 3017.

5. Song, X., Zhuang, Y., Lan, Y., Lin, Y., and Min, X. Comprehensive Review and Comparison for Anticancer Peptides Identification Models. Current protein \& peptide science 2020.

6. Brown, K.L., and Hancock, R.E. Cationic host defense (antimicrobial) peptides. Current opinion in immunology 2006; 18(1): 24-30.

7. Hancock, R.E., Haney, E.F., and Gill, E.E. The immunology of host defence peptides: beyond antimicrobial activity. Nature reviews. Immunology 2016; 16(5):321-34.

8. Mulder, K., Lima, L.A., Miranda, V., Dias, S.C., and Franco, O.L. Current scenario of peptide-based drugs: the key roles of cationic antitumor and antiviral peptides. Frontiers in microbiology 2013; 4:321.

9. Patathananone, S., Thammasirirak, S., Daduang, J., Chung, J.G., Temsiripong, Y., and Daduang, S. Bioactive compounds from crocodile (Crocodylus siamensis) white blood cells induced apoptotic cell death in hela cells. Environmental toxicology 2016; 31(8): 986-97.

10. Sousa, L.Q., Machado, K.D., Oliveira, S.F., Araujo, L.D., Moncao-Filho, E.D., Melo-Cavalcante, A.A., et al. Bufadienolides from amphibians: A promising source of anticancer prototypes for radical innovation, apoptosis triggering and $\mathrm{Na}(+) / \mathrm{K}(+)$-ATPase inhibition. Toxicon : official journal of the International Society on Toxinology 2017; 127: 63-76. 
11. Buchau, A.S., Morizane, S., Trowbridge, J., Schauber, J., Kotol, P., Bui, J.D., et al. The host defense peptide cathelicidin is required for NK cell-mediated suppression of tumor growth. J Immunol 2010; 184(1):369-78.

12. Piktel, E., Niemirowicz, K., Wnorowska, U., Watek, M., Wollny, T., Gluszek, K., et al. The Role of Cathelicidin LL-37 in Cancer Development. Archivum immunologiae et therapiae experimentalis 2016; 64(1):33-46.

13. Bergman, P., Walter-Jallow, L., Broliden, K., Agerberth, B., and Soderlund, J. The antimicrobial peptide LL-37 inhibits HIV-1 replication. Current HIV research 2007; 5(4): 410-5.

14. Tripathi, S., Verma, A., Kim, E.J., White, M.R., and Hartshorn, K.L. LL-37 modulates human neutrophil responses to influenza A virus. Journal of leukocyte biology 2014; 96(5): 931-8.

15. Sainz, B., Jr., Alcala, S., Garcia, E., Sanchez-Ripoll, Y., Azevedo, M.M., Cioffi, M., et al. Microenvironmental hCAP-18/LL-37 promotes pancreatic ductal adenocarcinoma by activating its cancer stem cell compartment. Gut 2015; 64(12):1921-35.

16. Ren, S.X., Cheng, A.S., To, K.F., Tong, J.H., Li, M.S., Shen, J., et al. Host immune defense peptide LL-37 activates caspase-independent apoptosis and suppresses colon cancer. Cancer research 2012; 72(24): 6512-23.

17. Shanmugam, G., Mohan, A., Kumari, K., Louis, J.M., Soumya Krishnan, U., Balagopal, P.G., et al. A novel reporter construct for screening small molecule inhibitors that specifically target self-renewing cancer cells. Exp Cell Res 2019; 383(2): 111551.

18. Shen, Y., Maupetit, J., Derreumaux, P., and Tuffery, P. Improved PEP-FOLD Approach for Peptide and Miniprotein Structure Prediction. Journal of chemical theory and computation 2014; 10(10): 4745-58.

19. Vasanwala, F.H., Kusam, S., Toney, L.M., and Dent, A.L. Repression of AP-1 function: a mechanism for the regulation of Blimp-1 expression and B lymphocyte differentiation by the B cell lymphoma- 6 protooncogene. J Immunol 2002; 169(4): 1922-9.

20. Shyla, G., Vineethkumar, T., Arun, V., Divya, M., Thomas, S., and George, S. Functional characterization of two novel peptides and their analogs identified from the skin secretion of Indosylvirana aurantiaca, an endemic frog species of Western Ghats, India. Chemoecology 2019: 1-9.

21. Zhang, D., Zhou, Q., Huang, D., He, L., Zhang, H., Hu, B., et al. ROS/JNK/c-Jun axis is involved in oridonin-induced caspase-dependent apoptosis in human colorectal cancer cells. Biochemical and biophysical research communications 2019; 513(3): 594-601.

22. Oh, K., Lee, O.Y., Park, Y., Seo, M.W., and Lee, D.S. IL-1beta induces IL-6 production and increases invasiveness and estrogen-independent growth in a TG2-dependent manner in human breast cancer cells. BMC cancer 2016; 16(1): 724 . 
23. Hassan, W., Ding, L., Gao, R.Y., Liu, J., and Shang, J. Interleukin-6 signal transduction and its role in hepatic lipid metabolic disorders. Cytokine 2014; 66(2): 133-42.

24. Van der Velden, J., Janssen-Heininger, Y.M., Mandalapu, S., Scheller, E.V., Kolls, J.K., and Alcorn, J.F. Differential requirement for c-Jun $\mathrm{N}$-terminal kinase 1 in lung inflammation and host defense. PloS one 2012; 7(4): e34638.

25. Decker, T., and Kovarik, P. Serine phosphorylation of STATs. Oncogene 2000; 19(21): 2628-37.

26. Patocka, J., Nepovimova, E., Klimova, B., Wu, Q., and Kuca, K. Antimicrobial Peptides: Amphibian Host Defense Peptides. Current medicinal chemistry 2018.

27. Swithenbank, L., Cox, P., Harris, L.G., Dudley, E., Sinclair, K., Lewis, P., et al. Temporin A and Bombinin H2 Antimicrobial Peptides Exhibit Selective Cytotoxicity to Lung Cancer Cells. Scientifica 2020; 2020: 3526286.

28. Shaheen, F., Nadeem-Ul-Haque, M., Ahmed, A., Simjee, S.U., Ganesan, A., Jabeen, A., et al. Synthesis of breast cancer targeting conjugate of temporin-SHa analog and its effect on pro- and anti-apoptotic protein expression in MCF-7 cells. Peptides 2018; 106: 68-82.

29. Wang, C., Zhou, Y., Li, S., Li, H., Tian, L., Wang, H., et al. Anticancer mechanisms of temporin-1CEa, an amphipathic alpha-helical antimicrobial peptide, in Bcap-37 human breast cancer cells. Life sciences 2013; 92(20-21): 1004-14.

30. Riedl, S., Zweytick, D., and Lohner, K. Membrane-active host defense peptides-challenges and perspectives for the development of novel anticancer drugs. Chemistry and physics of lipids 2011; 164(8): 766-81.

31. Keating, N., and Nicholson, S.E. SOCS-mediated immunomodulation of natural killer cells. Cytokine 2019; 118: 64-70.

32. Cendrowski, J., Mamińska, A., and Miaczynska, M. Endocytic regulation of cytokine receptor signaling. Cytokine \& growth factor reviews $2016 ; 32: 63-73$.

33. Zhang, J., Sun, Y., Kang, Y., and Shang, D. Antimicrobial peptide temporin-1CEa isolated from frog skin secretions inhibits the proinflammatory response in lipopolysaccharide-stimulated RAW264.7 murine macrophages through the MyD88-dependent signaling pathway. Molecular immunology $2021 ; 132$ : $227-$ 35.

34. Dong, W., Zhu, X., Zhou, X., Yang, Y., Yan, X., Sun, L., et al. Potential role of a series of lysine-/leucinerich antimicrobial peptide in inhibiting lipopolysaccharide-induced inflammation. The Biochemical journal 2018; 475(22):3687-706. 
35. Bezzerri, V., Avitabile, C., Dechecchi, M.C., Lampronti, I., Borgatti, M., Montagner, G., et al. Antibacterial and anti-inflammatory activity of a temporin $B$ peptide analogue on an in vitro model of cystic fibrosis. Journal of peptide science : an official publication of the European Peptide Society 2014; 20(10): 822-30.

36. Kurosaka, M., and Machida, S. Interleukin-6-induced satellite cell proliferation is regulated by induction of the JAK 2/STAT 3 signalling pathway through cyclin D1 targeting. Cell proliferation 2013; 46(4): 365-73.

37. Hirano, T., Ishihara, K., and Hibi, M. Roles of STAT3 in mediating the cell growth, differentiation and survival signals relayed through the IL-6 family of cytokine receptors. Oncogene 2000; 19(21): 2548.

38. Rios, P., Nunes-Xavier, C.E., Tabernero, L., Kohn, M., and Pulido, R. Dual-specificity phosphatases as molecular targets for inhibition in human disease. Antioxidants \& redox signaling 2014; 20(14): 2251-73.

39. Lang, R., and Raffi, F.A.M. Dual-Specificity Phosphatases in Immunity and Infection: An Update. International journal of molecular sciences $2019 ; 20(11)$.

40. Mei, Z.Z., Chen, X.Y., Hu, S.W., Wang, N., Ou, X.L., Wang, J., et al. Kelch-like Protein 21 (KLHL21) Targets IkappaB Kinase-beta to Regulate Nuclear Factor kappa-Light Chain Enhancer of Activated B Cells (NF-kappaB) Signaling Negatively. The Journal of biological chemistry 2016; 291(35): 18176-89.

41. Deng, Y., Ren, X., Yang, L., Lin, Y., and Wu, X. A JNK-dependent pathway is required for TNFalphainduced apoptosis. Cell 2003; 115(1):61-70.

42. Dhanasekaran, D.N., and Reddy, E.P. JNK signaling in apoptosis. Oncogene 2008; 27(48): 6245-51.

43. Hong, S.S., Choi, J.H., Lee, S.Y., Park, Y.H., Park, K.Y., Lee, J.Y., et al. A Novel Small-Molecule Inhibitor Targeting the IL-6 Receptor beta Subunit, Glycoprotein 130. J Immunol 2015; 195(1): 237-45.

44. Eghtedar, A., Verstovsek, S., Estrov, Z., Burger, J., Cortes, J., Bivins, C., et al. Phase 2 study of the JAK kinase inhibitor ruxolitinib in patients with refractory leukemias, including postmyeloproliferative neoplasm acute myeloid leukemia. Blood 2012; 119(20): 4614-8.

45. Seo, H.R., Kwan, Y.W., Cho, C.K., Bae, S., Lee, S.J., Soh, J.W., et al. PKCalpha induces differentiation through ERK1/2 phosphorylation in mouse keratinocytes. Experimental \& molecular medicine 2004; 36(4): 292-9.

46. Tanaka, T., Narazaki, M., Masuda, K., and Kishimoto, T. Regulation of IL-6 in Immunity and Diseases. Advances in experimental medicine and biology 2016; 941: 79-88.

47. Johnson, D.E., O'Keefe, R.A., and Grandis, J.R. Targeting the IL-6/JAK/STAT3 signalling axis in cancer. Nature reviews. Clinical oncology 2018; 15(4): 234-48. 
48. Boulanger, M.J., Chow, D.C., Brevnova, E.E., and Garcia, K.C. Hexameric structure and assembly of the interleukin-6/IL-6 alpha-receptor/gp130 complex. Science 2003; 300(5628): 2101-4.

49. de Hon, F.D., Klaasse Bos, H.K., Ebeling, S.B., Grotzinger, J., Kurapkat, G., Rose-John, S., et al. Leucine58 in the putative 5 th helical region of human interleukin (IL)- 6 is important for activation of the IL-6 signal transducer, gp130. FEBS letters 1995; 369(2-3): 187-91.

\section{Supplementary Tables And Figures}

The Supplementary Tables and Figures are not available with this version.

\section{Figures}




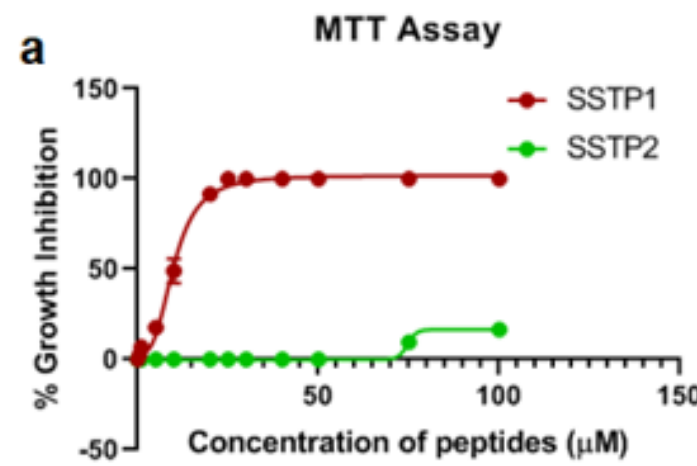

b
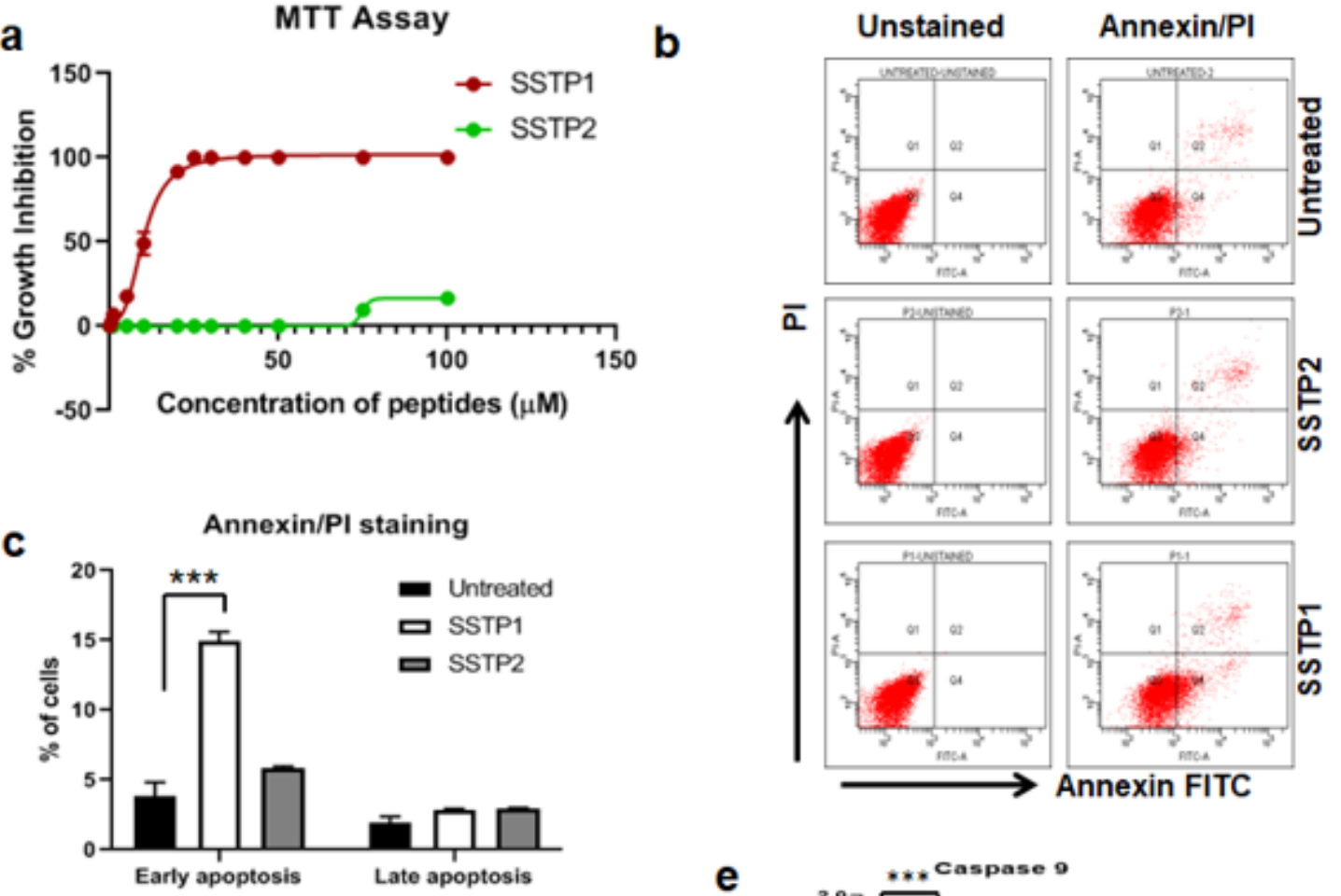

d

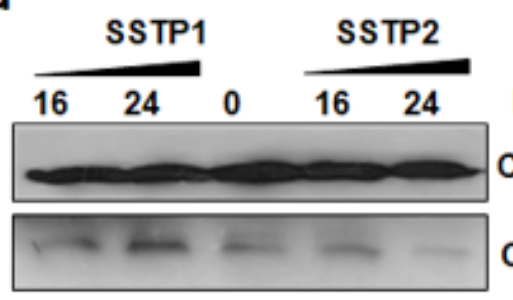

Hours

Casp 9 p47

e

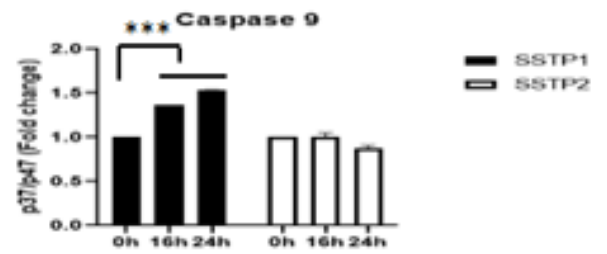

Casp 9 p37/35

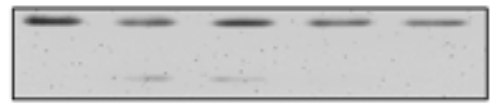

Casp 8 p57

Casp 8 p43/41

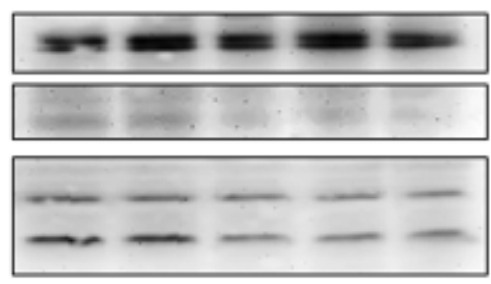

Casp 3 p35

Casp 3 p19/17

Casp 7 p28

Casp 7 p20

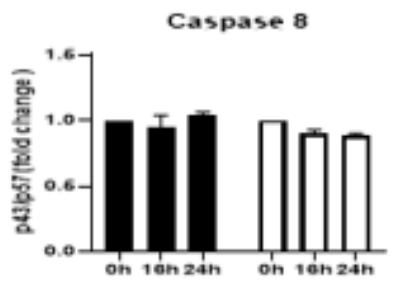

ש EsTP1

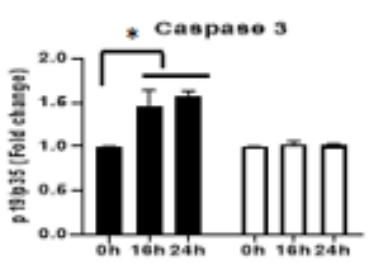

D eotpi

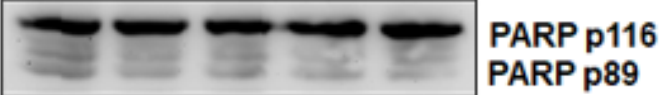

PARP p89

$\beta$-Actin

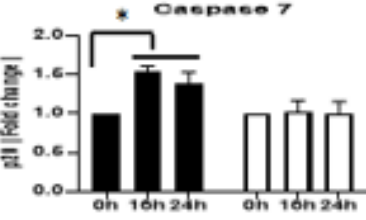

\section{Figure 1}

SSTP1 induces apoptosis in oral cancer cells (a) HSC-4 cells were treated with peptides SSTP1 and SSTP2 at different concentrations in $0.5 \%$ DMEM for $48 \mathrm{~h}$, and MTT assay was performed to calculate \% growth inhibition. The graph was plotted using GraphPad Prism8.2.1 using non-linear four parameter curve fit. The error bars indicate SEM of three biological replicates (b) HSC-4 cells were treated with 10 $\mu \mathrm{M}$ SSTP1 and SSTP2 for 22h and FACS analysis was performed after annexin/PI staining. (c)The 
quantified populations are represented in the graph. The error bars indicate standard deviation of triplicates. (d) The HSC-4 cells treated with $10 \mu \mathrm{M}$ SSTP1 or SSTP2 for indicated time intervals were used for western blot analyses (e) Densitometric analysis was done for the bands, and fold change based on Omin was calculated. The error bars indicate standard deviation of biological replicates.

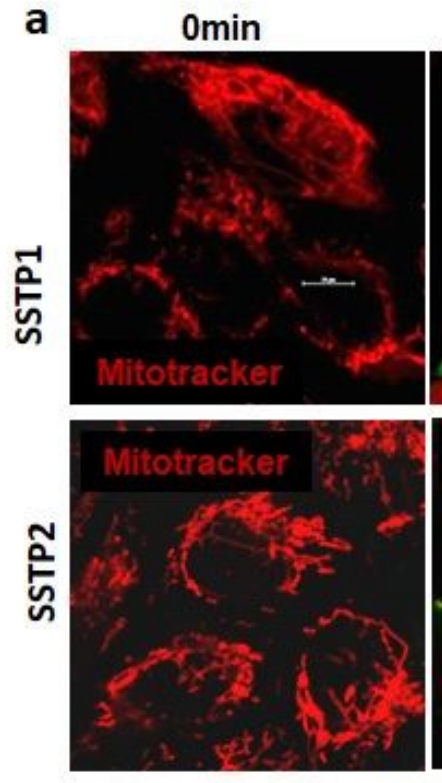

$37^{\circ} \mathrm{C}$

b
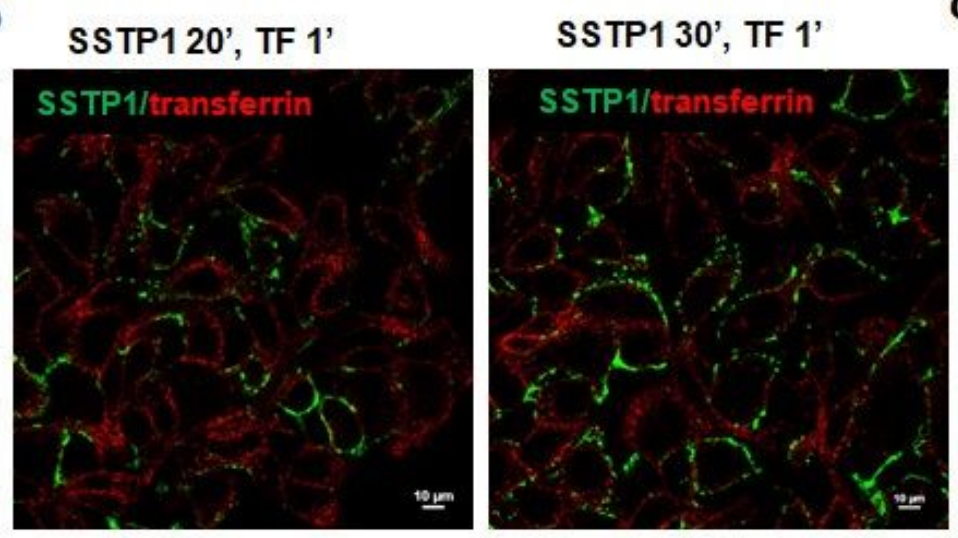
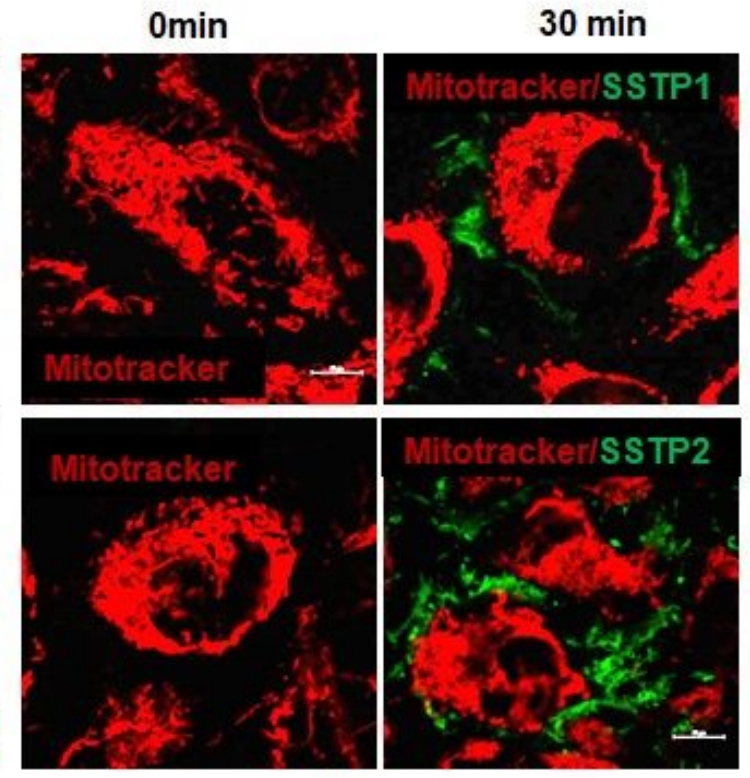

$0^{\circ} \mathrm{C}$

C SSTP1 20', TF 3'

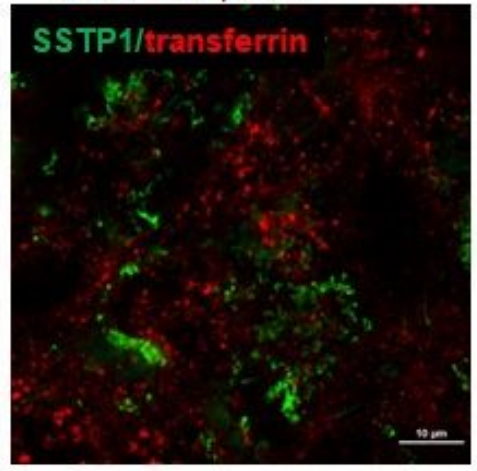

d

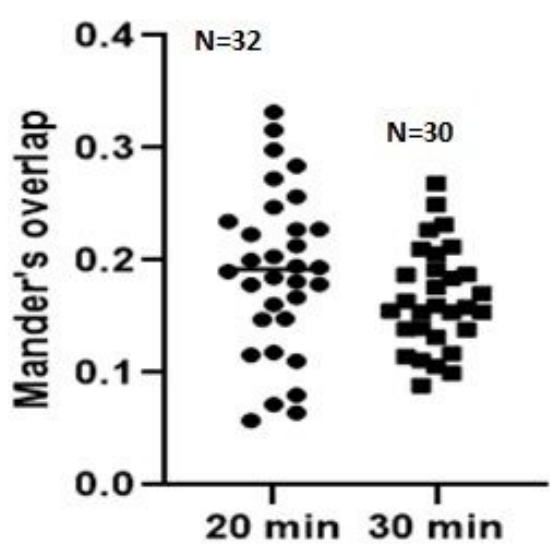

Figure 2 
SSTP1 is internalized by active uptake. Live HSC-4 cells were incubated with MitoTracker Deep Red and incubated for $10 \mathrm{~min}$. Excess dye was washed off, and then the respective peptides were added, which were labeled with Alexa Fluor-488, and incubated either on ice or at $37^{\circ} \mathrm{C}$. Images were acquired at the indicated time intervals. (b) the cells were treated with Alexa Fluor-488-labelled SSTP1 for indicated time and Alexa Fluor-568-labelled transferrin was added and incubated for one minute. The cells were washed, fixed and imaged. (c) The cells were treated with SSTP1-Alexa Fluor-488 for 20 min, transferrin-568 was added to it and incubated for one minute. Cells were washed to remove unbound SSTP1 and transferrin. Live imaging was performed after 2 minutes. (d) The extent of co-occurrence for panel b was measured and plotted as Manderson's overlap. The median value is shown in the graph. 
a Transcripts

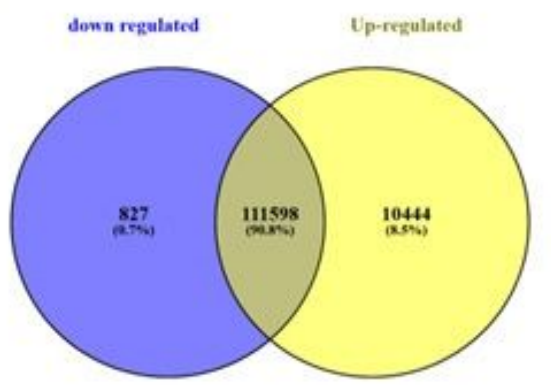

b Genes

C

\begin{tabular}{|c|c|c|c|c|c|c|}
\hline SSTP2 SSTP & & SSTP2 SSTP1 & & SSTP2 & SSTP1 & \\
\hline- & $R R A D$ & $\Rightarrow 0$ & JUN & $=$ & $\Rightarrow$ & BCL11 A \\
\hline$c=0$ & PTGS2 & -2 & SOCS1 & & & \\
\hline$-m$ & NR4A1 & $\ldots-$ & KLFG & & & SOX2 \\
\hline $\mathrm{m}^{2} \mathrm{x}$ & NFATC2 & $=0$ & FOSB & $=$ & $m . \infty$ & TP73 \\
\hline - & RHOB & - & CD55 & 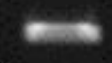 & nomise & $B M F$ \\
\hline & & & FOSLI & & & \\
\hline
\end{tabular}

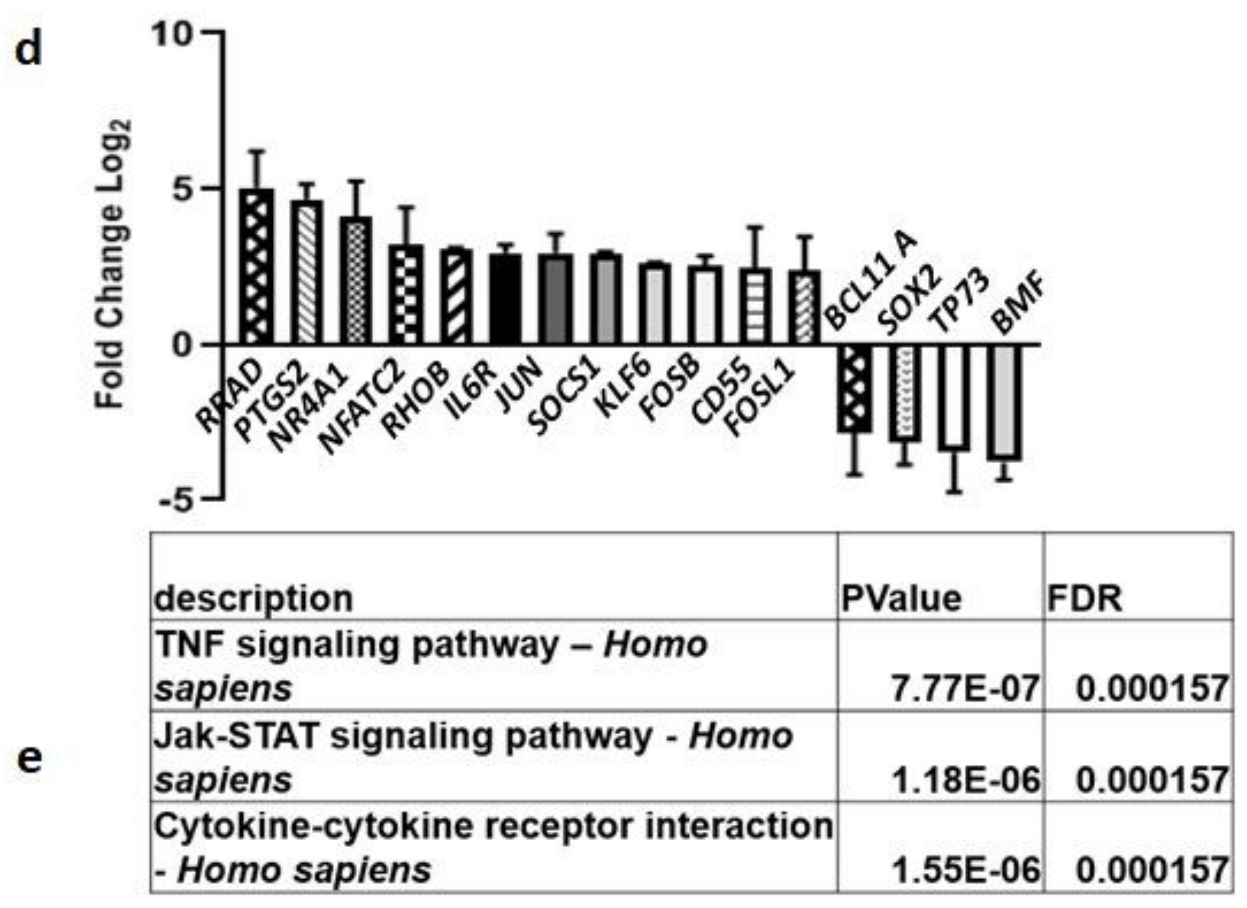

Figure 3

RNA-Seq analysis suggests the activation of cytokine signaling pathway upon SSTP1 treatment. (a) HSC4 cells were treated with $10 \mu \mathrm{M}$ SSTP1 and SSTP2 for 3h, and total RNA was isolated to perform RNASeq analysis. The Venn diagram represents the differential expression of transcripts upon SSTP1 treatment compared to SSTP2-treatment. (b) Volcano plot showing differential expression profile of genes showing significant up regulation and down regulations on treatment with SSTP1 with respect to 
control peptide SSTP2. Red dots indicate absolute log 2 fold change $\geq 2$ and FDR-adjusted $p$ value $\leq 0.05$ and blue dots indicate log 2 fold change $\geq 2$ and $p$ value $\leq 0.05$. (c, d) The results were confirmed by RTPCR and qPCR for the indicated molecules. (e) A pathway enrichment analysis was performed based on the differential gene expression analysis, and top 3 pathways that were regulated are tabulated.

a
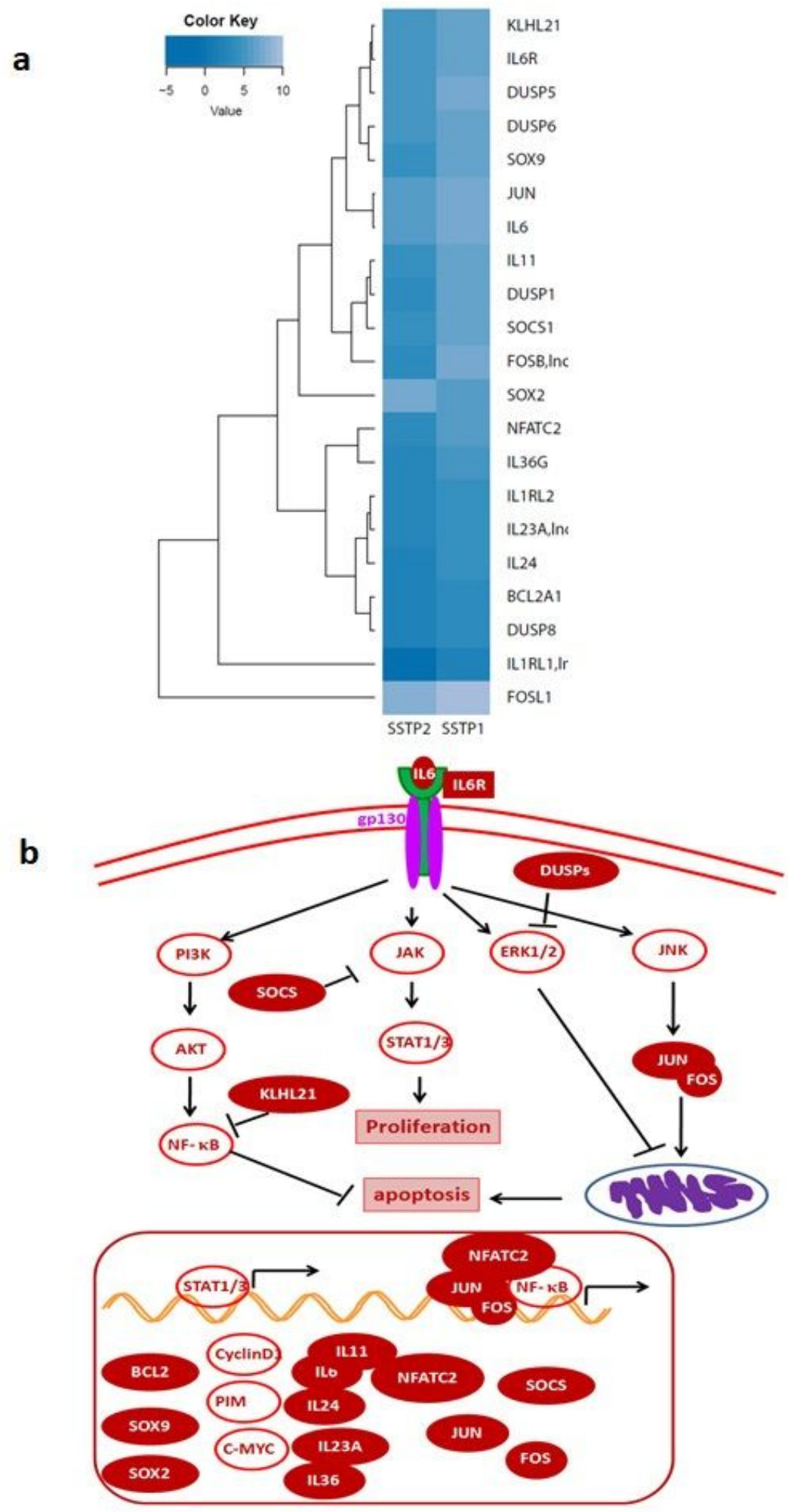

Figure 4 
Cytokine signaling pathway involved in SSTP1 response (a) STAT/.JAK, JNK/AP1 MAPK1/2, and AKT pathways are reported to be downstream of Cytokine signaling. Heat map showing differential expression of molecules involved in cytokine signaling are shown. (b) Schematic diagram of cytokine signaling pathway in SSTP1 response.
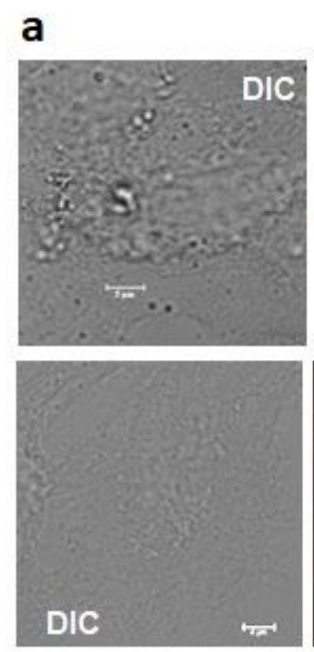

c
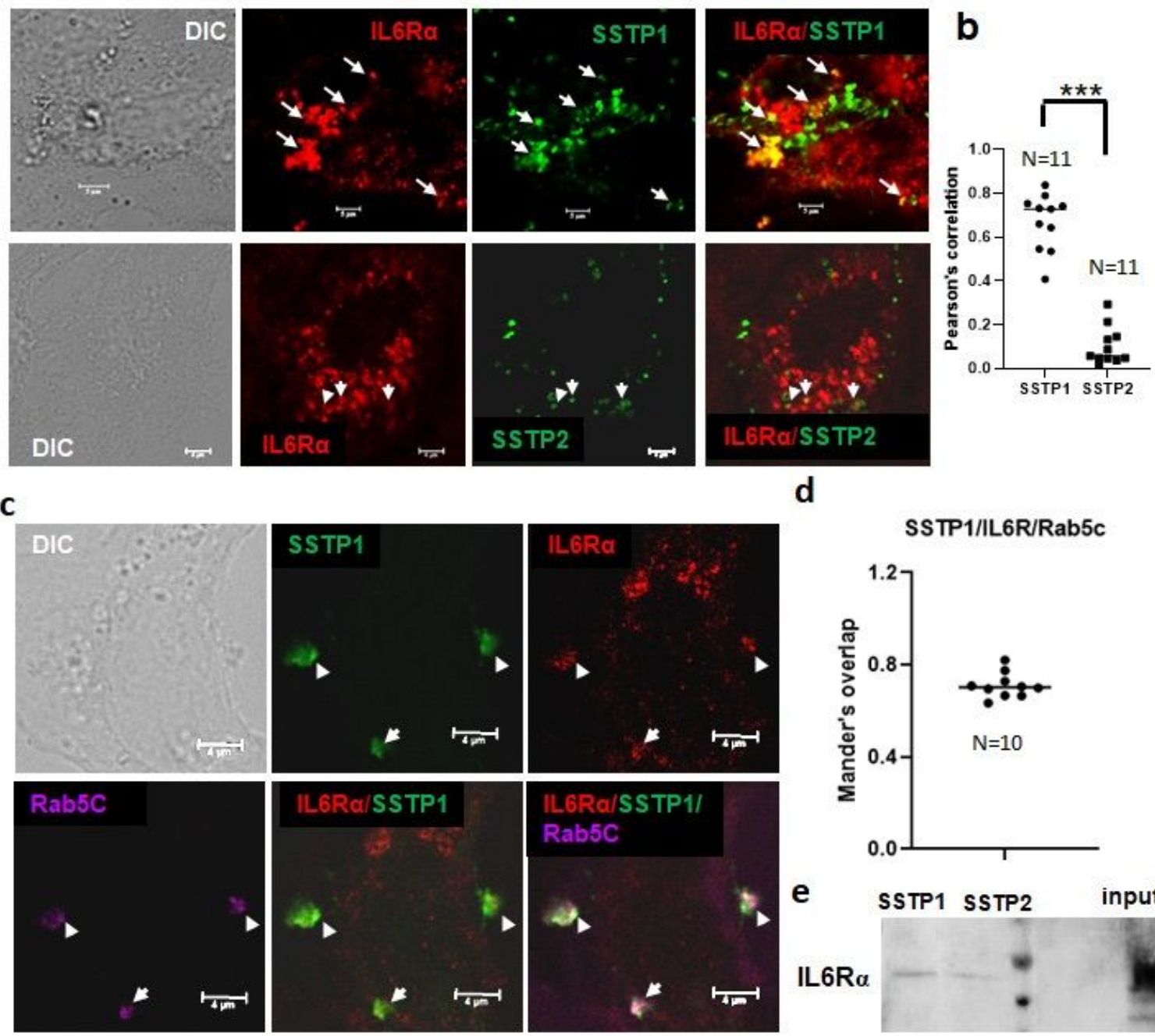

d
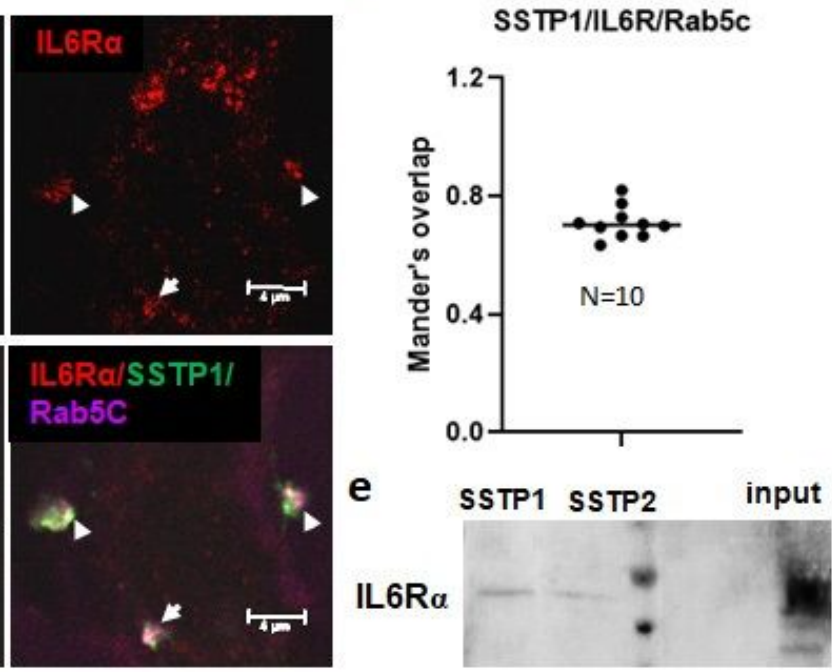

f

SSTP1-Alexa Fluor-488

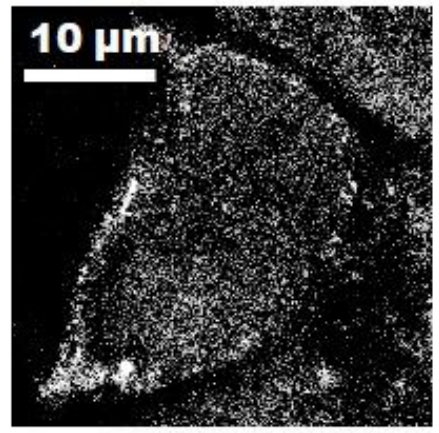

FRET

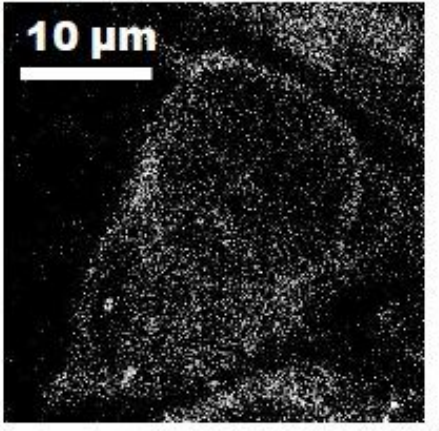

\section{IL6R $\alpha$-Alexa Fluor-555}

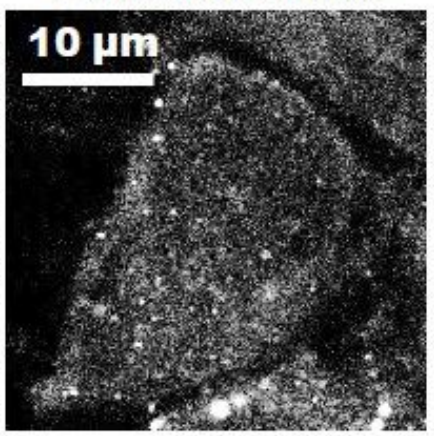

Figure 5 
SSTP1 interacts with IL6Ra and mediate the uptake of SSTP1 (a) HSC-4 cells were treated with Biotin labeled-SSTP1 or SSTP2 $(10 \mu \mathrm{M})$ for 30 minutes and then fixed and probed without permeabilization using mouse IL6Ra (2B2.3). (b) The co-localization of SSTP1 or SSTP2 to IL6Ra was analyzed for 11 cells, and the Pearson's correlation was plotted. (c) Cells were treated as in panel a and fixed and permeabilized to probe for IL6Ra, SSTP1 and Rab5C. The arrow mark indicates co-localization. The scale bars represent $4 \mu \mathrm{m}$ (d) The Mander's overlap in SSTP1 foci were quantified for 10 cells. The average value for each cell is plotted (e) Pull-down assay using SSTP1-biotin or SSTP2-biotin and probed for IL6Ra.(f) FRET analysis to show the SSTP1 binding to IL6Ra as described in Methods. 
a
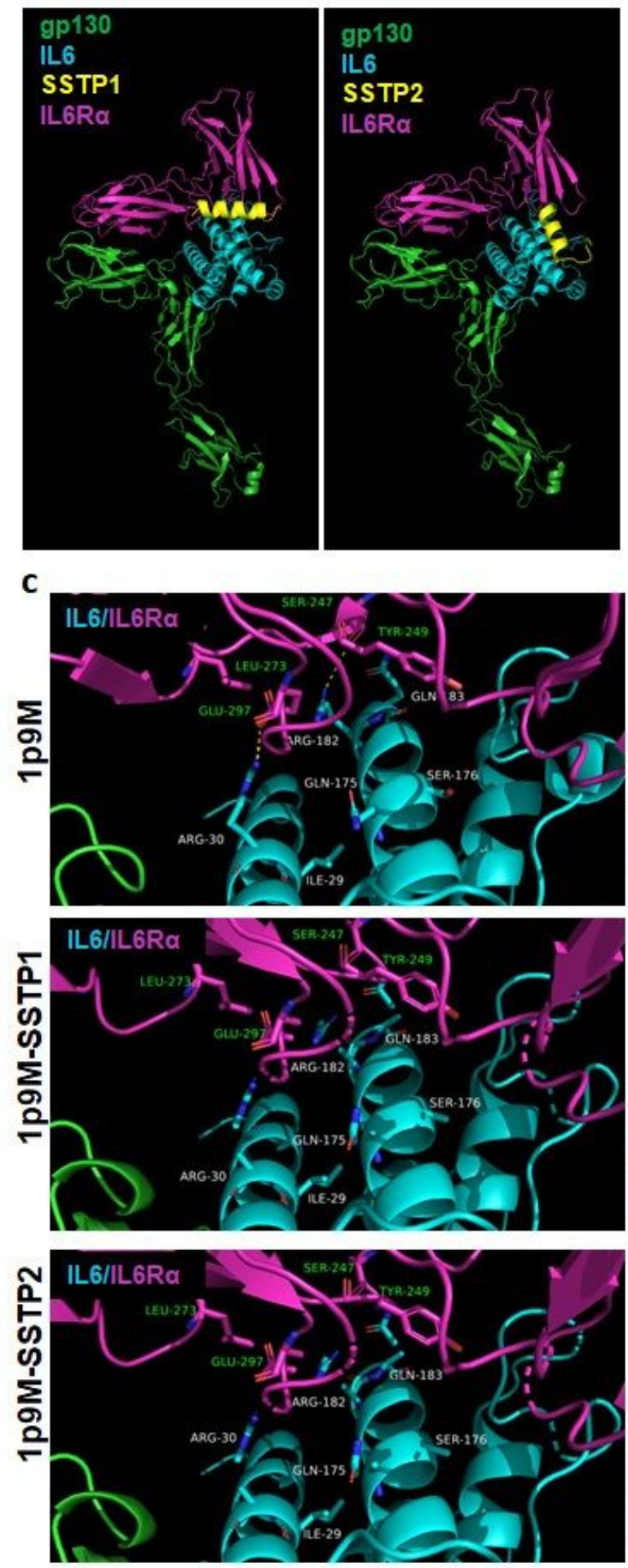

b
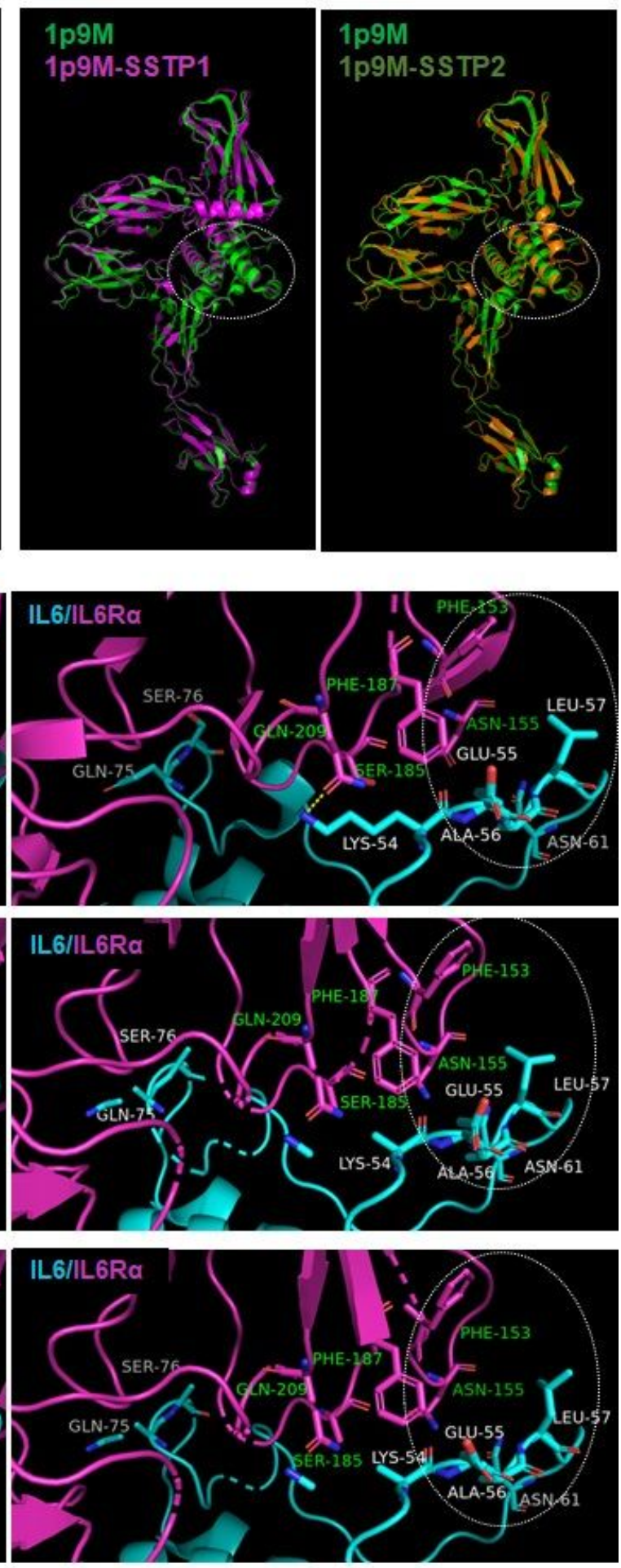

\section{Figure 6}

Binding of SSTP1 and SSTP2 to IL6/IL6Ra/gp130 complex alters the active site interactions (a) The predicted structure of SSTP1 and SSTP2 was docked to the crystal structure of trimeric complex 1p9M, and the stable structures identified were obtained after dynamic simulation. (b) the SSTP1 or SSTP2bound active trimeric complex was superimposed to $1 \mathrm{p} 9 \mathrm{M}$. The white circle represents the site with unique changes. (c) High resolution images of the interacting residues of IL6 and IL6Ra. The residues of 
IL6 chain is marked using white font color and the green font represents the residues of IL6Ra. The circle with dotted line denotes the site with unique changes.

\section{Image not available with this version}

\section{Figure 7}

SSTP1 preferentially activates JNK/AP1 pathway (a) HSC-4 cells were treated with the peptides as indicated before for the indicated time points, and the lysates were used for western blot. (b) Densitometric analysis was performed and relative phosphorylation of each molecule was calculated. Fold change based on untreated from biological duplicates were plotted. (c) A luciferase reporter assay was performed using 3×AP1pGL3 reporter construct after treatment with the peptides $(10 \mu \mathrm{M})$ for $48 \mathrm{~h}$. The beetle luciferase activity was normalized with renilla luciferase activity. 


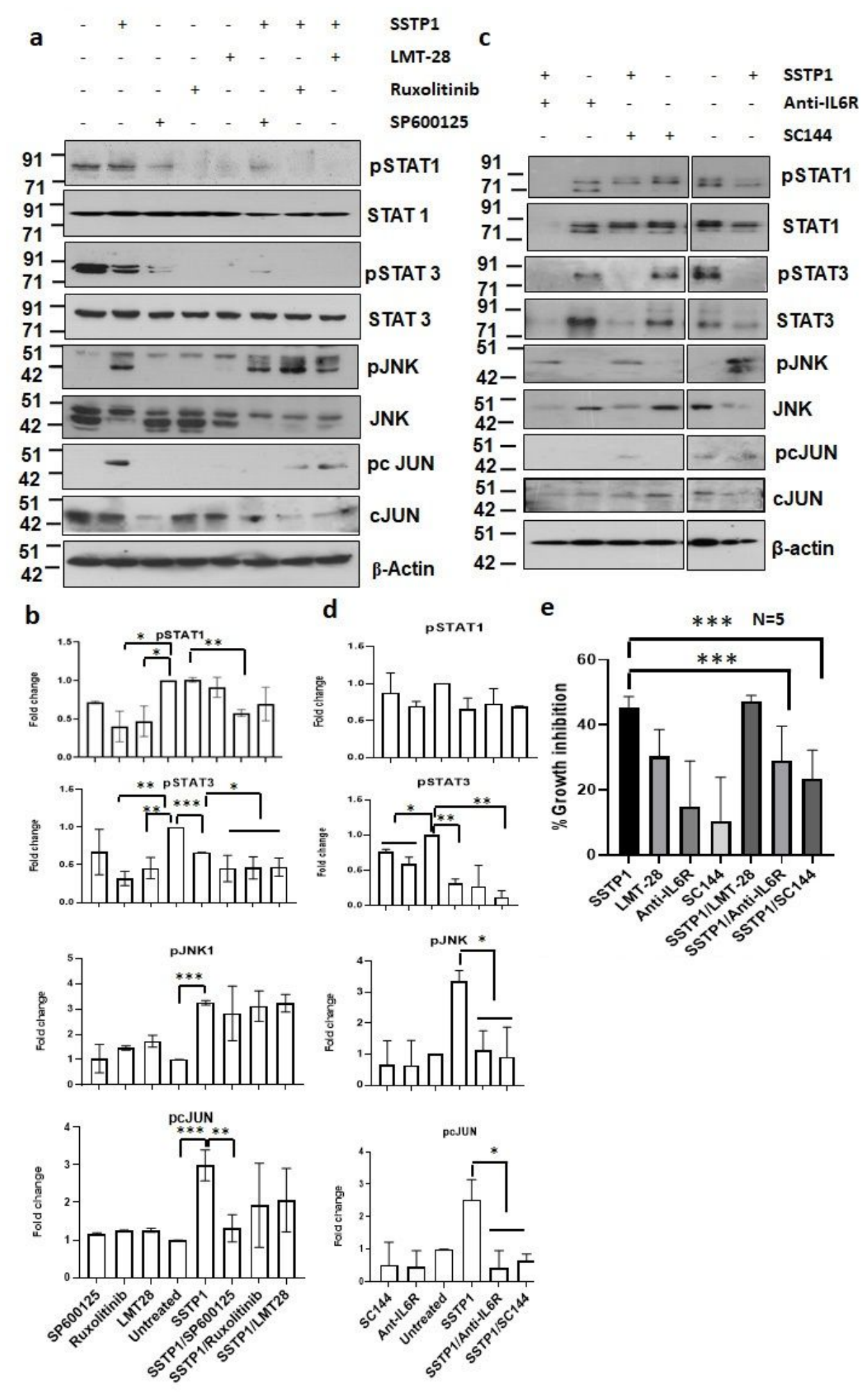

Figure 8

SSTP1-induced pcJUN activation depends on JNK and on gp130 and IL6Ra. (a) HSC-4 cells treated with the peptides and inhibitors were used for western blot analysis of the indicated molecules (b)

Densitometric analysis was done for the bands of (a) and relative phosphorylation is plotted. (c) The cells treated with the peptides and IL6R $a$ antibody and gp130 inhibitors were used for western blot analysis of the indicated molecules. (d) Densitometric analysis was done for the bands of (c) and relative 
a

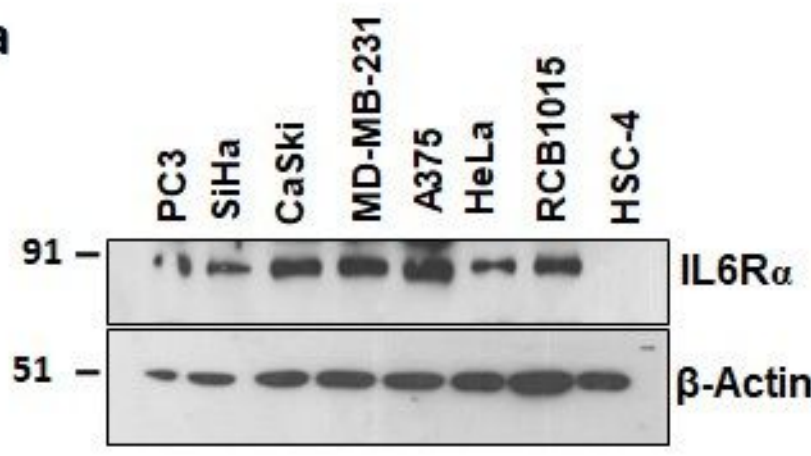

C

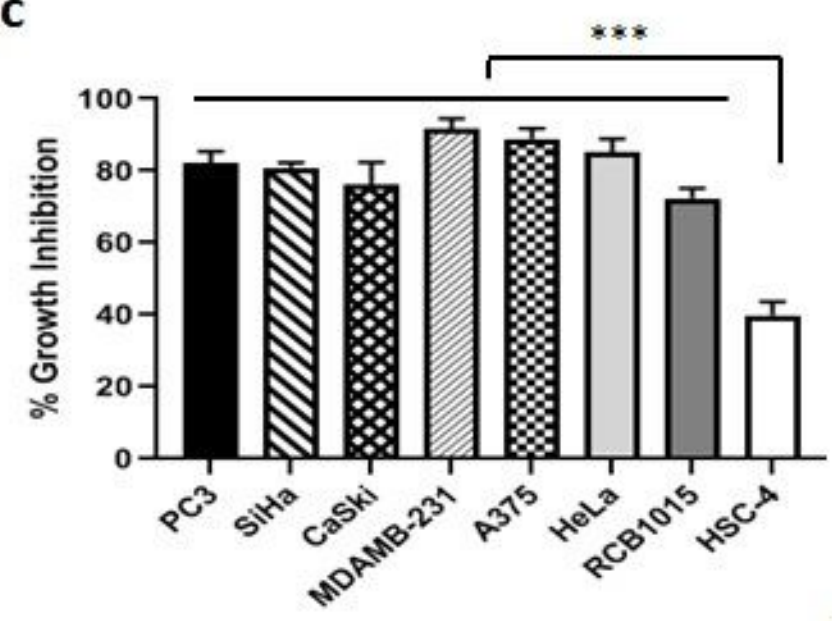

e

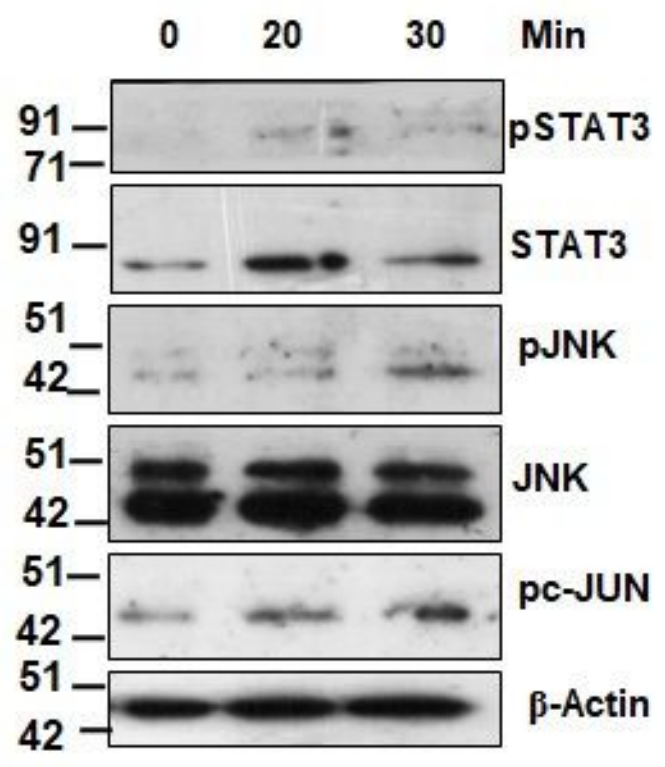

b
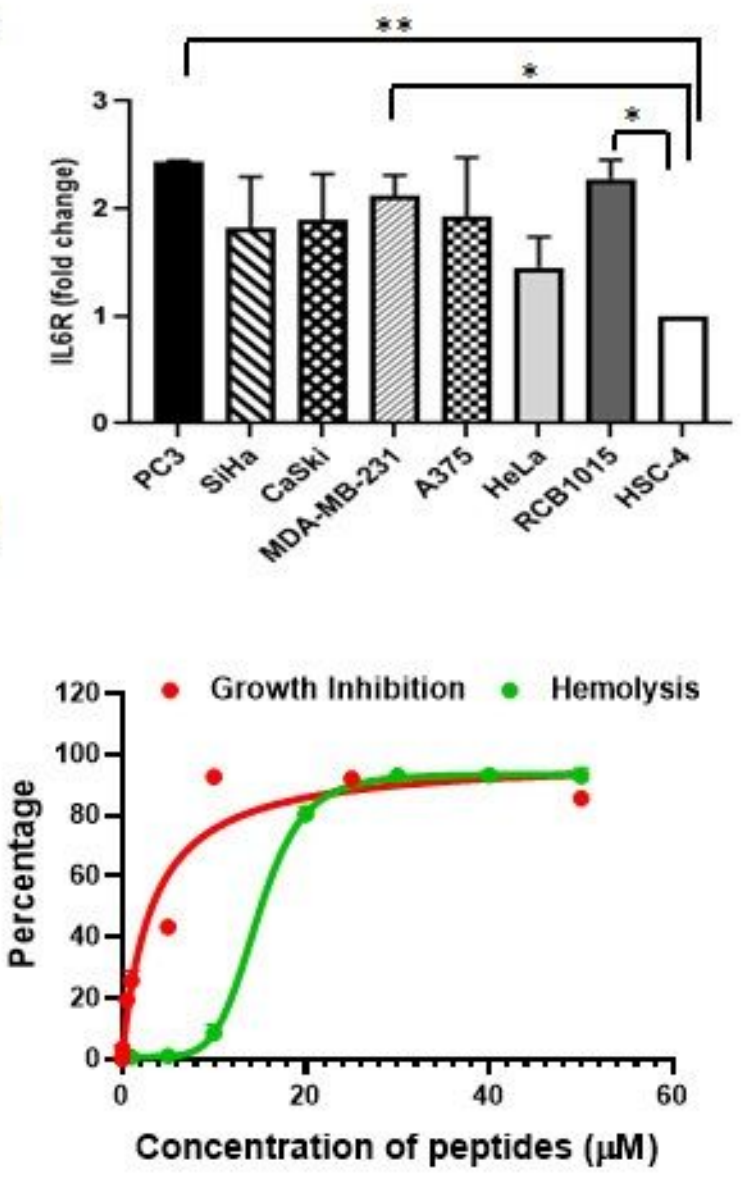

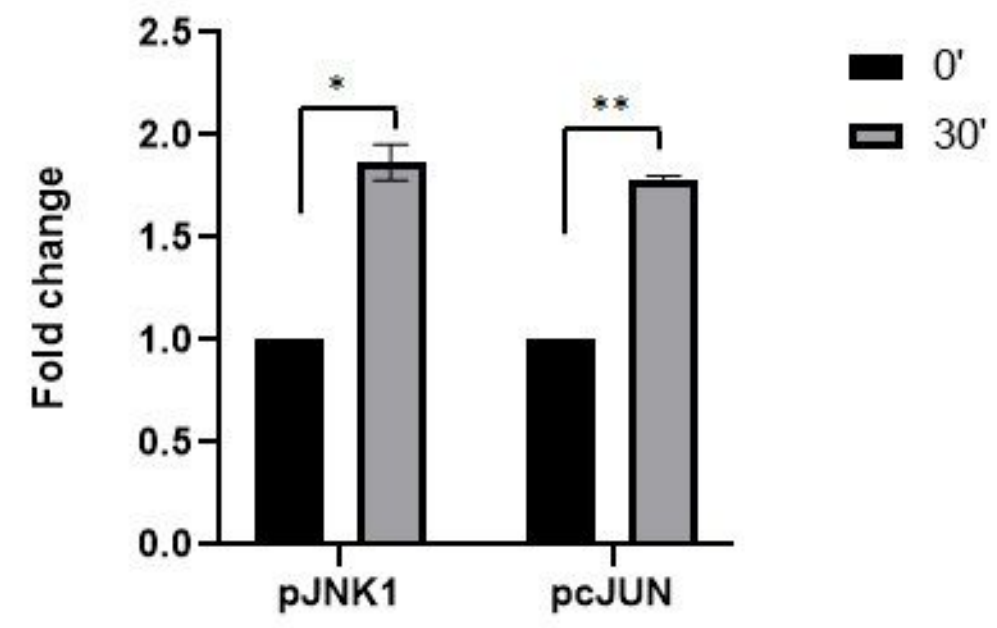

Figure 9

Sensitivity of cancer cells to SSTP1 depends on the level of IL6Ra. (a) Western blot performed using cell lysates of different cancer cells. (b) The densitometric analysis of the bands of the blot using ImageJ. (c) Growth inhibition analyzed by MTT assay using $10 \mu \mathrm{M}$ SSTP1 treated for 48h. (d) Growth inhibitory curve 
of SSTP1 for MDA-MB-231 cells analyzed after 48h treatment with SSTP1 and Hemolysis induced by SSTP1 on human RBC. The error bars indicate SEM of three biological replicates (e) MDA-MB-231 cells were treated with $4.5 \mu \mathrm{M}$ SSTP1 for the indicated time intervals and probed for the indicated molecules by western blot $(f)$ The blots were quantified using ImageJ, and the graphs were plotted 